\title{
Synthesis of 1,4-Dichloro-1,3-butadienes by Rhodium-Complex-Catalyzed Reaction of Terminal Alkynes with Trichloroacetyl Chloride
}

Taigo Kashiwabara, Kouichirou Fuse, Takeshi Muramatsu and Masato Tanaka*

Chemical Resources Laboratory, Tokyo Institute of Technology, 4259 Nagatsuta, Midori-ku, Yokohama 226-8503, Japan

m.tanaka@res.titech.ac.jp

Table of Contents

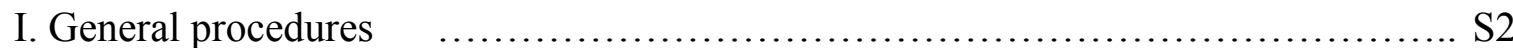

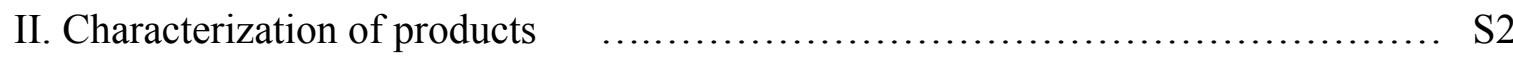

III. Formation and reaction of rhodium complexes relevant to the mechanism $\quad \ldots . . . \mathrm{S} 3$

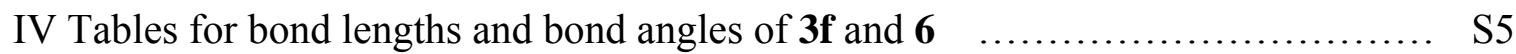

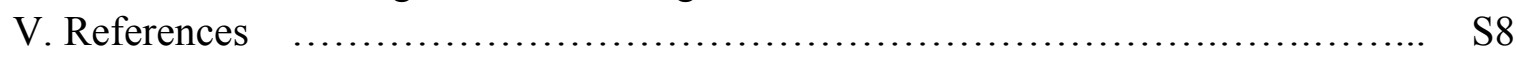

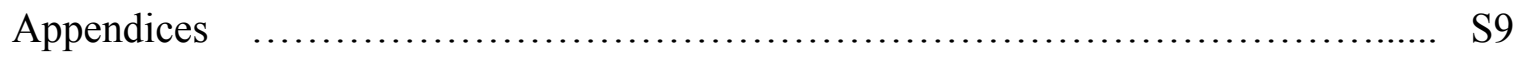




\section{General Procedures}

All manipulations involving air and moisture-sensitive compounds were carried out under atmosphere of dry argon or nitrogen, by using standard Schlenk tube techniques. All solvents were distilled over appropriate drying agents prior to use. ${ }^{1} \mathrm{H}$ NMR $(300 \mathrm{MHz}),{ }^{13} \mathrm{C}\left\{{ }^{1} \mathrm{H}\right\}$ NMR $(75$ $\mathrm{MHz}),{ }^{29} \mathrm{Si}\left\{{ }^{1} \mathrm{H}\right\} \mathrm{NMR}(60 \mathrm{MHz})$ and ${ }^{31} \mathrm{P}\left\{{ }^{1} \mathrm{H}\right\} \mathrm{NMR}(162 \mathrm{MHz})$ spectra were recorded at ambient temperature, using $\mathrm{CDCl}_{3}$ as solvent unless otherwise noted. ${ }^{1} \mathrm{H}$ and ${ }^{13} \mathrm{C}\left\{{ }^{1} \mathrm{H}\right\}$ NMR chemical shifts were reported in ppm relative to internal $\mathrm{Me}_{4} \mathrm{Si} .{ }^{29} \mathrm{Si}\left\{{ }^{1} \mathrm{H}\right\} \mathrm{NMR}$ chemical shift was reported in ppm relative to internal $\mathrm{Me}_{4} \mathrm{Si} .{ }^{31} \mathrm{P}\left\{{ }^{1} \mathrm{H}\right\} \mathrm{NMR}$ chemical shifts were reported in ppm relative to external aq $85 \% \mathrm{H}_{3} \mathrm{PO}_{4}$. GC was run using a column (SUS column $2.0 \mathrm{~mm} \phi \times 2.0 \mathrm{~m}$ ) packed with silicone OV17 5\% on Chromosorb W (60/80 mesh). GCMS was run at an ionization potential of $70 \mathrm{eV}$. High resolution mass spectra were obtained at an ionization potential of $70 \mathrm{eV}$.

1-Octyne, 1-decyne, tert-butylacetylene, 5-chloro-1-pentyne, 5-hexynonitrile, methyl 5-hexynoate, trimethylsilylacetylene, 3-phenyl-1-propyne, phenylacetylene, p-methoxyphenylacetylene, p-tolylacetylene, p-fluorophenylacetylene, 1-ethynylcyclohexene, ethynylferrocene, 4-octyn, methyl propiolate, trichloroacetyl chloride) and triphenylarsine were obtained from commercial source and distilled/recrystallized prior to use. Ethynylthiophene, ${ }^{1}$ 4-(tert-butyldimethylsiloxy)-1-butyne, ${ }^{2}\left[\mathrm{RhCl}(\mathrm{CO})_{2}\right]_{2},{ }^{3}[\mathrm{Rh}(\text { acac })(\mathrm{CO})]_{2},{ }^{4}$ tribromoacetyl bromide, ${ }^{5}$ tris[(3,5-dimesityl)phenyl]phosphine ${ }^{6}$ and authentic samples of fac,cis- $\mathrm{RhCl}_{3}(\mathrm{CO})\left(\mathrm{PMe}_{3}\right)_{2}$ and mer,trans- $\mathrm{RhCl}_{3}(\mathrm{CO})\left(\mathrm{PMe}_{3}\right)_{2}{ }^{7}$ were prepared according to the literature.

To estimate the ${ }^{1} \mathrm{H}$ NMR yield of 3a, intensity of the olefinic proton signal was quantified with reference to that of the proton signal arising from 1,1,2,2-tetrachloroethane used as internal standard. The program for ${ }^{1} \mathrm{H}$ NMR measurement was set as follows: pulse $=30^{\circ}$, acquisition time $=2.65 \mathrm{sec}$, pulse delay $=3.65 \mathrm{sec}$.

\section{Characterization of products}

Products $\mathbf{3 b}, \mathbf{3 i}, \mathbf{3 k}$ and 4 , documented in the literature ${ }^{8}$ were identified by comparison of the spectroscopic data with those reported and products $\mathbf{3 c}, \mathbf{3} \mathbf{j}$ and $\mathbf{3 l}$ were fully characterized with satisfactory elemental analysis. The other products $\mathbf{3 a}, \mathbf{3 d}, \mathbf{3 e}, \mathbf{3 f}, \mathbf{3 g}, \mathbf{3 h}$ and $\mathbf{3 m}$ displayed satisfactory spectroscopic data, including HRMS, but without satisfactory elemental analysis. For ${ }^{1} \mathrm{H}$ and ${ }^{13} \mathrm{C}$ NMR spectra of 3a, 3c, 3d, 3e, 3f, 3g, 3h, 3j, $3 \mathbf{l}$ and 3m, see Appendixes $1-20 .{ }^{1} \mathrm{H}$ and ${ }^{13} \mathrm{C}$ NMR spectra of $\mathbf{3 b}, \mathbf{3} \mathbf{i}$ and $\mathbf{3 k}$ are also included in Supporting Information as Appendixes 21-26. Structures of several byproducts were characterized by GC and GCMS, the latter of which are also listed below.

\section{An isomer of $(Z, Z)-1,4,7,10$-tetrachlorodeca-4,6-diene (3c)}

GCMS analysis of the reaction of 5-chloro-1-pentyne suggested that a trace ( $<1 \%$ yield) of an isomer of $(Z, Z)-1,4,7,10$-tetrachlorodeca-4,6-diene (3c) appeared to have been formed. GCMS (EI, $70 \mathrm{eV}) \mathrm{m} / \mathrm{z}$ (\% relative intensity) $276\left(\mathrm{M}^{+}, 87\right), 239$ (24), 211 (32), 175 (20), 167 (35), 139 (100), 113 (19), 103 (30).

\section{X-Ray analysis of (Z,Z)-1,6-diphenyl-2,5-dichloro-2,4-hexadiene (3f)}

Single crystals were obtained by recrystallization from a $\mathrm{CH}_{2} \mathrm{Cl}_{2} /$ hexane $(99 / 1)$ solution. A yellow crystal $(=0.25 \times 0.30 \times 0.50 \mathrm{~mm})$ was used for X-ray diffraction data collection on a diffractometer with $\mathrm{MoK} \alpha$ radiation $\left(=0.7107 \AA\right.$ ). $\mathrm{C}_{9} \mathrm{H}_{8} \mathrm{Cl}, \mathrm{M}=156.65$, triclinic, Pnma(\#62), $\mathrm{a}=$ 
5.684(4) $\AA, \mathrm{b}=8.214(5) \AA, \mathrm{c}=8.368(5) \AA, \alpha=71.04(2)^{\circ}, \beta=82.51(2)^{\circ}, \gamma=82.59(2)^{\circ}, \mathrm{V}=$ $364.8(4) \AA^{3}, Z=2, \mathrm{D}($ calc. $)=1.426 \mathrm{~g} / \mathrm{cm}^{3}$. Total reflection collected $=2476, \mathrm{GOF}=0.984, R(\mathrm{I}>$ $2.00 \sigma(\mathrm{I}))=0.035, R w(\mathrm{I}>2.00 \sigma(\mathrm{I})$, all reflections $)=0.053$. Selected bond distances and angles are summarized in Tables S1-1 and S1-2.

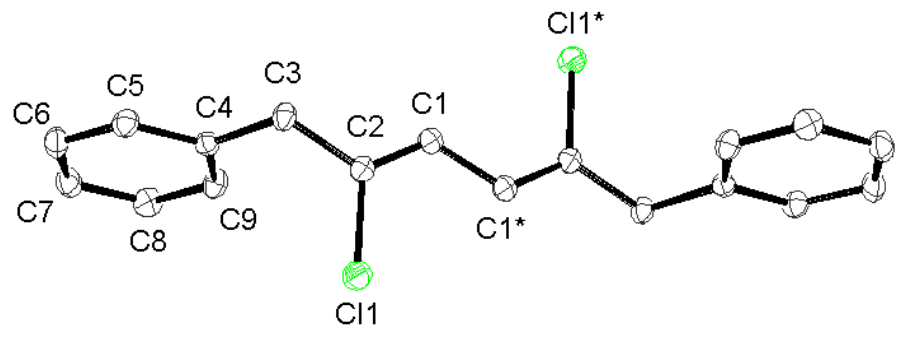

Figure S1. Molecular structure of 3f. The hydrogen atoms are omitted for clarity and the thermal ellipsoids are drawn at the $30 \%$ probability level.

\section{Reaction of methyl propiolate}

The reaction run as in the representative reaction procedure did not form the desired product, but only oligomers appeared to be formed on the basis of the following GCMS analysis.

Dimer of methyl propiolate: GCMS (EI) m/z (\% relative intensity) $168\left(\mathrm{M}^{+}, 3\right), 155$ (13), 154 (100), $152(7)$.

Trimer of methyl propiolate, isomer A: GCMS (EI) m/z (\% relative intensity) $252\left(\mathrm{M}^{+}\right.$, 7), 222 (12), 221 (100), 194 (2), 191 (2).

Trimer of methyl propiolate, isomer B: GCMS (EI) $\mathrm{m} / \mathrm{z}$ (\% relative intensity) $252\left(\mathrm{M}^{+}\right.$, 34), 222 (14), 221 (100), 193 (7).

\section{Reaction of tribromoacetyl bromide with 1-decyne}

The reaction was run and analyzed similarly to the representative reaction procedure to reveal the formation of (Z,Z)-9,12-dibromoicosa-9,11-diene 4 in $41 \%$ NMR yield. GC and GCMS analyses appeared to suggest that large quantities of isomeric 1,2-dibromo-1-decenes were also formed as byproducts, but no effort was made to quanify them.

\section{1,2-Dibromo-1-decene}

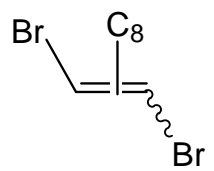

Isomer 1: GCMS (EI, $70 \mathrm{eV}) \mathrm{m} / \mathrm{z}\left(\%\right.$ relative intensity) $298\left(\mathrm{M}^{+}, 6\right), 199(10), 137(32)$, 119 (10), 95 (77), 81 (100), 69 (41).

Isomer 2: GCMS (EI, $70 \mathrm{eV}) \mathrm{m} / \mathrm{z}$ (\% relative intensity) $298\left(\mathrm{M}^{+}, 8\right), 199$ (4), 137 (25), 119 (10), 95 (84), 81 (100), 69 (26).

\section{Formation and reaction of rhodium complexes relevant to the mechanism}

\section{General procedure for the reaction of $\operatorname{RhCl}(\mathrm{CO})\left(\mathrm{PMe}_{3}\right)_{2}$ with trichloroacetyl chloride in $\mathrm{CDCl}_{3}$}

The procedure is exemplified by the experiment run at $0{ }^{\circ} \mathrm{C}$, which was as follows. To a 
yellow solution of $\mathrm{RhCl}(\mathrm{CO})\left(\mathrm{PMe}_{3}\right)_{2}(10.0 \mathrm{mg}, 0.0314 \mathrm{mmol})$ in $\mathrm{CDCl}_{3}(0.45 \mathrm{~mL})$ kept at $0{ }^{\circ} \mathrm{C}$ was added trichloroacetyl chloride $(3.5 \mu \mathrm{L}, 0.0314 \mathrm{mmol})$. The color changed near instantaneously to red. After $15 \mathrm{~min}$ at the temperature, the mixture was analyzed ${ }^{31} \mathrm{P} \mathrm{NMR}$ spectroscopy to display a major doublet at $-1.27 \mathrm{ppm}\left({ }^{1} J_{\mathrm{Rh}-\mathrm{P}}=72.8 \mathrm{~Hz}\right)$ assignable to fac,cis- $\mathrm{RhCl}_{3}(\mathrm{CO})\left(\mathrm{PMe}_{3}\right)_{2}$ (fac,cis-5) and a minor doublet at $-0.91 \mathrm{ppm}\left({ }^{1} J_{\mathrm{Rh}-\mathrm{P}}=76.3 \mathrm{~Hz}\right)$, together with two more even minor doublets at -2.32 and $-3.78 \mathrm{ppm}$..

\section{Catalytic reaction using a material obtained by treating $\mathrm{RhCl}_{3}$ with two equivalents of $\mathbf{P}(\mathbf{o}-\mathrm{Tol})_{3}$.}

$\mathrm{RhCl}_{3}(4.3 \mathrm{mg}, 0.021 \mathrm{mmol}), \mathrm{P}(o-\mathrm{Tol})_{3}(12.2 \mathrm{mg}, 0.0400 \mathrm{mmol})$ and ethanol $(1.0 \mathrm{~mL})$ were mixed in a $20 \mathrm{~mL}$ Schlenk tube to develop a pink slurry in $3 \mathrm{~min}$, which was heated for 30 min to give a yellow solution. Evaporation of ethanol left yellow solid. ${ }^{31} \mathrm{P}$ NMR measurement in $\mathrm{CDCl}_{3}$ indicated that the material contained paramagnetic species at least partially and displayed broad signals at $50.8,-22.1 \mathrm{ppm}$ in addition to another signal at $40.0 \mathrm{ppm}$, which could be assigned to $\mathrm{O}=\mathrm{P}(\mathrm{o}-\mathrm{Tol})_{3}{ }^{9}$ No signal was found $-27.9 \mathrm{ppm}$ due to free $\mathrm{P}(o-\mathrm{Tol})_{3} .{ }^{10}$ Although we do understood that the material was not pure, we used it as such in a catalytic reaction under the standard conditions $\left(130{ }^{\circ} \mathrm{C}, 12 \mathrm{~h}\right.$, in ethylbenzene), since the color of our material was totally different from $\mathrm{RhCl}_{2}\left[\mathrm{P}(\mathrm{o}-\mathrm{Tol})_{3}\right]_{2}$, reported by Bennett. ${ }^{11}$ Analysis of the resulting mixture showed $100 \%$ conversion of 1 -octyne (GC) and $28 \%$ yield of $3 \mathbf{a}\left({ }^{1} \mathrm{H}\right.$ NMR). 
IV. Tables for bond lengths and bond angles of $3 f$ and 6

Table S1-1. Bond lengths ( $\AA$ ) of (Z,Z)-1,6-diphenyl-2,5-dichloro-2,4-hexadiene (3f)

$\begin{array}{llllll}\text { atom } & \text { atom } & \text { distance } & \text { atom } & \text { atom } & \text { distance } \\ \mathrm{Cl}(1) & \mathrm{C}(2) & 1.7480(17) & \mathrm{C}(1) & \mathrm{C}(1)^{*} & 1.449(2) \\ \mathrm{C}(1) & \mathrm{C}(2) & 1.338(2) & \mathrm{C}(2) & \mathrm{C}(3) & 1.497(2) \\ \mathrm{C}(3) & \mathrm{C}(4) & 1.522(2) & \mathrm{C}(4) & \mathrm{C}(5) & 1.390(2) \\ \mathrm{C}(4) & \mathrm{C}(9) & 1.385(2) & \mathrm{C}(5) & \mathrm{C}(6) & 1.393(2) \\ \mathrm{C}(6) & \mathrm{C}(7) & 1.378(3) & \mathrm{C}(7) & \mathrm{C}(8) & 1.390(2) \\ \mathrm{C}(8) & \mathrm{C}(9) & 1.386(2) & & & \end{array}$

Table S1-2. Bond angles $\left(^{\circ}\right)$ of of (Z,Z)-1,6-diphenyl-2,5-dichloro-2,4-hexadiene (3f)

$\begin{array}{llllllll}\text { atom } & \text { atom } & \text { atom } & \text { angle } & \text { atom } & \text { atom } & \text { atom } & \text { angle } \\ \mathrm{C}(1)^{*} & \mathrm{C}(1) & \mathrm{C}(2) & 126.33(15) & \mathrm{Cl}(1) & \mathrm{C}(2) & \mathrm{C}(1) & 120.93(13) \\ \mathrm{Cl}(1) & \mathrm{C}(2) & \mathrm{C}(3) & 114.22(11) & \mathrm{C}(1) & \mathrm{C}(2) & \mathrm{C}(3) & 124.85(15) \\ \mathrm{C}(2) & \mathrm{C}(3) & \mathrm{C}(4) & 114.96(15) & \mathrm{C}(3) & \mathrm{C}(4) & \mathrm{C}(5) & 119.12(17) \\ \mathrm{C}(3) & \mathrm{C}(4) & \mathrm{C}(9) & 122.07(14) & \mathrm{C}(5) & \mathrm{C}(4) & \mathrm{C}(9) & 118.75(17) \\ \mathrm{C}(4) & \mathrm{C}(5) & \mathrm{C}(6) & 120.44(19) & \mathrm{C}(5) & \mathrm{C}(6) & \mathrm{C}(7) & 120.25(16) \\ \mathrm{C}(6) & \mathrm{C}(7) & \mathrm{C}(8) & 119.69(18) & \mathrm{C}(7) & \mathrm{C}(8) & \mathrm{C}(9) & 119.85(19) \\ \mathrm{C}(4) & \mathrm{C}(9) & \mathrm{C}(8) & 121.00(15) & & & & \end{array}$


Tables S2-1. Bond lengths $(\AA)$ of $\left[\mathrm{Rh}(\mu-\mathrm{Cl}) \mathrm{Cl}_{2}\left(\mathrm{PMe}_{3}\right)_{2}\right]_{2}(\mathbf{6})$

$\begin{array}{llllll}\text { atom } & \text { atom } & \text { distance } & \text { atom } & \text { atom } & \text { distance } \\ \text { Rh1 } & \mathrm{Cl1} & 2.350(2) & \mathrm{Rh} 1 & \mathrm{Cl1}{ }^{*} & 2.350(2) \\ \mathrm{Rh} 1 & \mathrm{Cl} 2 & 2.371(2) & \mathrm{Rh} 1 & \mathrm{Cl}{ }^{*} & 2.371(2) \\ \mathrm{Rh} 1 & \mathrm{P} 1 & 2.331(4) & \mathrm{Rh} 1 & \mathrm{P} 2 & 2.351(4) \\ \mathrm{Rh} 2 & \mathrm{Cl} 2 & 2.493(2) & \mathrm{Rh} 2 & \mathrm{Cl}{ }^{*} & 2.493(2) \\ \mathrm{Rh} 2 & \mathrm{C} 3 & 2.328(4) & \mathrm{Rh} 2 & \mathrm{C} 14 & 2.336(4) \\ \mathrm{Rh} 2 & \mathrm{P} 3 & 2.256(3) & \mathrm{Rh} 2 & \mathrm{P}{ }^{*} & 2.256(3) \\ \mathrm{P} 1 & \mathrm{C} 1 & 1.804(13) & \mathrm{P} 1 & \mathrm{C}{ }^{*} & 1.804(13) \\ \mathrm{P} 1 & \mathrm{C} 2 & 1.793(15) & \mathrm{P} 2 & \mathrm{C} 3 & 1.777(15) \\ \mathrm{P} 2 & \mathrm{C} 4 & 1.804(13) & \mathrm{P} 2 & \mathrm{C}{ }^{*} & 1.804(13) \\ \mathrm{P} 3 & \mathrm{C} 5 & 1.771(13) & \mathrm{P} 3 & \mathrm{C} 6 & 1.93(2) \\ \mathrm{P} 3 & \mathrm{C} 7 & 1.91(2) & \mathrm{P} 3 & \mathrm{C} 8 & 1.78(2) \\ \mathrm{P} 3 & \mathrm{C} 9 & 1.73(2) & \mathrm{C} 6 & \mathrm{C} 9 & 0.89(4) \\ \mathrm{C} 7 & \mathrm{C} 8 & 0.96(4) & & & \end{array}$

Tables S2-2. Bond angles $\left(^{\circ}\right)$ of $\left[\mathrm{Rh}(\mu-\mathrm{Cl}) \mathrm{Cl}_{2}\left(\mathrm{PMe}_{3}\right)_{2}\right]_{2}(\mathbf{6})$

\begin{tabular}{|c|c|c|c|c|c|c|c|}
\hline atom & atom & atom & angle & atom & atom & atom & angle \\
\hline $\mathrm{Cl1}$ & Rh1 & $\mathrm{Cl}^{*}$ & $94.21(9)$ & $\mathrm{Cl} 1$ & Rh1 & $\mathrm{Cl} 2$ & $90.88(9)$ \\
\hline $\mathrm{Cl1}$ & Rh1 & $\mathrm{Cl} 2^{*}$ & $174.89(9)$ & $\mathrm{Cl1}$ & Rh1 & $\mathrm{P} 1$ & $87.06(10)$ \\
\hline $\mathrm{Cl1}$ & Rh1 & $\mathrm{P} 2$ & $87.42(10)$ & $\mathrm{Cl}^{*}$ & Rh1 & $\mathrm{Cl} 2$ & $174.89(9)$ \\
\hline $\mathrm{Cl1}^{*}$ & Rh1 & $\mathrm{Cl} 2^{*}$ & $90.88(9)$ & $\mathrm{Cl}^{*}$ & Rh1 & $\mathrm{P} 1$ & $87.06(10)$ \\
\hline $\mathrm{Cl1}^{*}$ & Rh1 & $\mathrm{P} 2$ & $87.42(10)$ & $\mathrm{Cl} 2$ & Rh1 & $\mathrm{Cl} 2^{*}$ & $84.02(9)$ \\
\hline $\mathrm{Cl} 2$ & Rh1 & $\mathrm{P} 1$ & $92.89(11)$ & $\mathrm{Cl} 2$ & Rh1 & $\mathrm{P} 2$ & $93.14(11)$ \\
\hline $\mathrm{Cl} 2^{*}$ & Rh1 & $\mathrm{P} 1$ & $92.89(11)$ & $\mathrm{Cl}^{*}$ & Rh1 & $\mathrm{P} 2$ & $93.14(11)$ \\
\hline $\mathrm{P} 1$ & Rh1 & $\mathrm{P} 2$ & $171.89(15)$ & $\mathrm{Cl} 2$ & Rh2 & $\mathrm{Cl} 2^{*}$ & $79.07(9)$ \\
\hline $\mathrm{Cl} 2$ & Rh2 & $\mathrm{Cl} 3$ & $90.12(11)$ & $\mathrm{Cl} 2$ & $\mathrm{Rh} 2$ & $\mathrm{Cl} 4$ & $90.84(11)$ \\
\hline $\mathrm{Cl} 2$ & Rh2 & P3 & $93.12(9)$ & $\mathrm{Cl} 2$ & Rh2 & $\mathrm{P} 3^{*}$ & $172.19(10)$ \\
\hline $\mathrm{Cl} 2^{*}$ & Rh2 & $\mathrm{Cl} 3$ & $90.12(11)$ & $\mathrm{Cl} 2^{*}$ & Rh2 & $\mathrm{Cl} 4$ & $90.84(11)$ \\
\hline $\mathrm{Cl} 2^{*}$ & $\mathrm{Rh} 2$ & P3 & $172.19(10)$ & $\mathrm{Cl} 2^{*}$ & $\mathrm{Rh} 2$ & $\mathrm{P} 3^{*}$ & $93.12(9)$ \\
\hline $\mathrm{Cl3}$ & Rh2 & $\mathrm{Cl} 4$ & $178.76(16)$ & $\mathrm{Cl} 3$ & $\mathrm{Rh} 2$ & P3 & $89.65(11)$ \\
\hline $\mathrm{Cl} 3$ & Rh2 & $\mathrm{P} 3^{*}$ & $89.65(11)$ & $\mathrm{Cl} 4$ & $\mathrm{Rh} 2$ & P3 & $89.52(10)$ \\
\hline $\mathrm{Cl} 4$ & Rh2 & $\mathrm{P}^{*}{ }^{*}$ & $89.52(10)$ & P3 & $\mathrm{Rh} 2$ & $\mathrm{P} 3^{*}$ & $94.68(11)$ \\
\hline Rh1 & $\mathrm{Cl} 2$ & Rh2 & $98.44(8)$ & Rh1 & $\mathrm{P} 1$ & $\mathrm{C} 1$ & $116.0(4)$ \\
\hline Rh1 & $\mathrm{P} 1$ & $\mathrm{C} 1^{*}$ & $116.0(4)$ & Rh1 & $\mathrm{P} 1$ & $\mathrm{C} 2$ & $111.7(6)$ \\
\hline $\mathrm{C} 1$ & $\mathrm{P} 1$ & $\mathrm{C} 1^{*}$ & $106.2(6)$ & $\mathrm{C} 1$ & $\mathrm{P} 1$ & $\mathrm{C} 2$ & $102.6(5)$ \\
\hline $\mathrm{C} 1{ }^{*}$ & P1 & $\mathrm{C} 2$ & $102.6(5)$ & Rh1 & $\mathrm{P} 2$ & $\mathrm{C} 3$ & $110.4(5)$ \\
\hline Rh1 & $\mathrm{P} 2$ & $\mathrm{C} 4$ & $116.8(4)$ & Rh1 & $\mathrm{P} 2$ & $\mathrm{C} 4^{*}$ & $116.8(4)$ \\
\hline $\mathrm{C} 3$ & $\mathrm{P} 2$ & $\mathrm{C} 4$ & $103.0(5)$ & $\mathrm{C} 3$ & $\mathrm{P} 2$ & $\mathrm{C} 4^{*}$ & $103.0(5)$ \\
\hline $\mathrm{C} 4$ & $\mathrm{P} 2$ & $\mathrm{C} 4^{*}$ & $105.2(5)$ & Rh2 & P3 & $\mathrm{C} 5$ & $111.4(5)$ \\
\hline $\mathrm{Rh} 2$ & P3 & C6 & $111.3(10)$ & $\mathrm{Rh} 2$ & P3 & $\mathrm{C} 7$ & $117.7(10)$ \\
\hline $\mathrm{Rh} 2$ & P3 & $\mathrm{C} 8$ & $117.8(8)$ & Rh2 & P3 & C9 & $122.0(11)$ \\
\hline $\mathrm{C} 5$ & P3 & C6 & $94.3(11)$ & $\mathrm{C} 5$ & P3 & $\mathrm{C} 7$ & $88.5(10)$ \\
\hline $\mathrm{C} 5$ & P3 & $\mathrm{C} 8$ & $113.7(9)$ & $\mathrm{C} 5$ & P3 & C9 & $109.7(12)$ \\
\hline C6 & P3 & $\mathrm{C} 7$ & $125.9(14)$ & C6 & P3 & $\mathrm{C} 8$ & $105.7(12)$ \\
\hline C6 & P3 & C9 & $27.5(13)$ & $\mathrm{C} 7$ & P3 & $\mathrm{C} 8$ & $29.9(12)$ \\
\hline
\end{tabular}




$\begin{array}{llllllll}\text { C7 } & \text { P3 } & \text { C9 } & 102.3(14) & \text { C8 } & \text { P3 } & \text { C9 } & 78.6(13) \\ \text { P3 } & \text { C6 } & \text { C9 } & 64(2) & \text { P3 } & \text { C7 } & \text { C8 } & 68(2) \\ \text { P3 } & \text { C8 } & \text { C7 } & 83(2) & \text { P3 } & \text { C9 } & \text { C6 } & 89(2)\end{array}$




\section{References}

1) Carpita, A.; Rossi, R.; Veracini, C. A. Tetrahedron 1985, 41, 1919.

2) Oila, M. J.; Tois, J. E.; Koskinen, A. M. P. Tetrahedron 2005, 61, 10748.

3) McCleverty, G. A.; Wilkinson, G.. Inorg. Synth. 1966, 8, 211.

4) Bonati, F.; Wilkinson, G. J. Chem. Soc. 1964, 3151.

5) Bogachev, V. S.; Ulanov, P. A. Russ. J. Bioorg. Chem. 2003, 29, 56.

6) Niyomura, O.; Iwasawa, T.; Sawada, N.; Tokunaga, M.; Obora, Y.; Tsuji, Y. Organometallics 2005, 24, 3468.

7) Browing, J.; Goggin, P. L.; Goodfellow, R. J.; Norton, M. G.; Rattray, A. J. M.; Taylor, B. F.; Mink, J. J. Chem. Soc., Dalton Trans. 1977, 2061.

8) Li, J.-H.; Liang, Y.; Xie, Y.-X. J. Org. Chem. 2004, 69, 8125.

9) Howell, J. A. S.; Palin, M. G.; Yates, P. C.; McArdle, P.; Cunningham, D.; Goldschmidt, Z.; Gottieb, H. E.; Hezroni-Langerman, D. J. Chem. Soc., Perkin Trans. 2 1992, 1769.

10) Ziegler, C. B.; Heck, R. F. J. Org. Chem. 1978, 43, 2941.

11) Bennett, M. A.; Longstaff, P. A. J. Am. Chem. Soc. 1969, 91, 6266. 
Appendix 1: ${ }^{1} \mathrm{H}$ NMR spectrum of (Z,Z)-7,10-dichlorohexadeca-7,9-diene (3a)
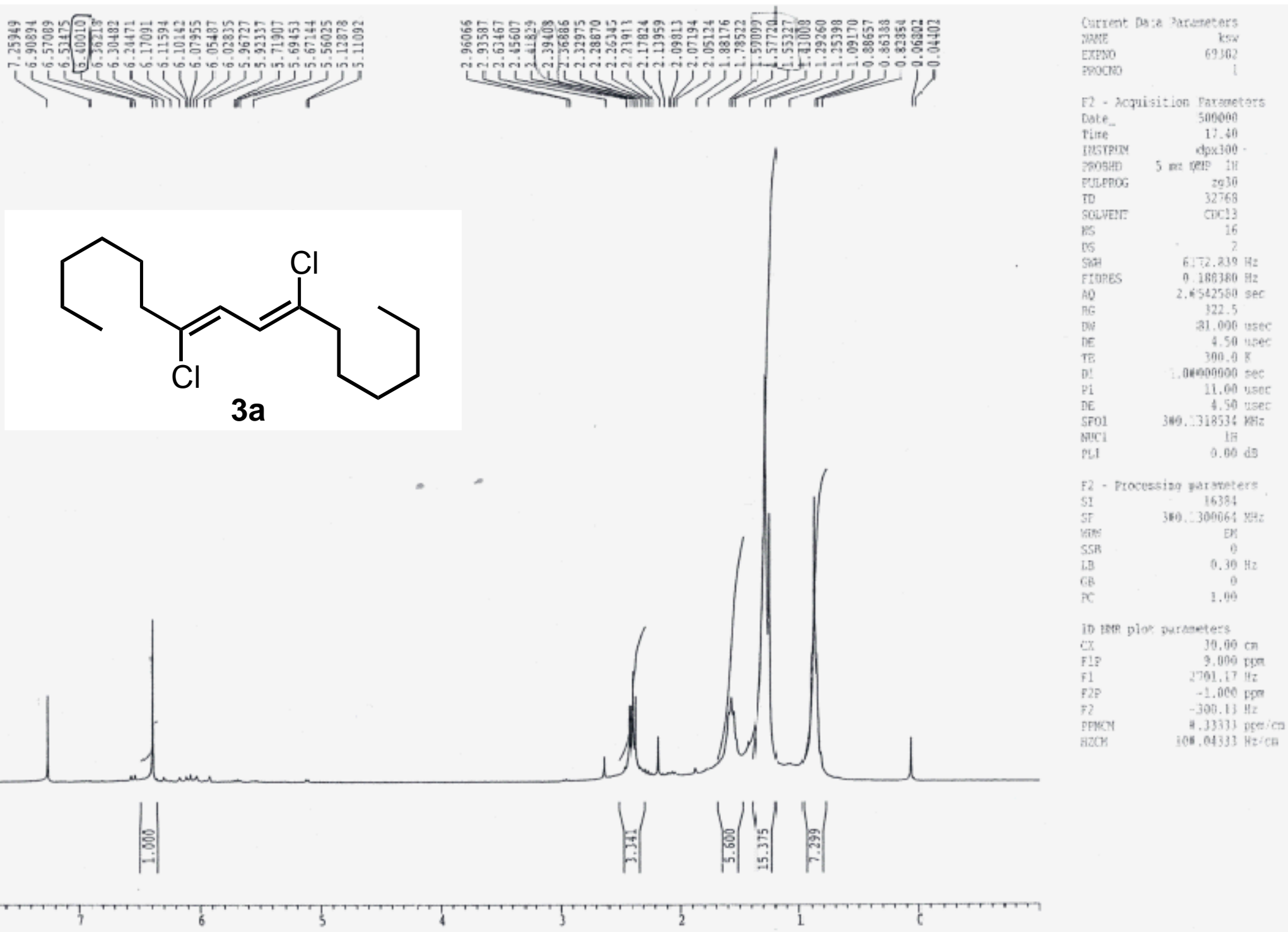

$3 a$

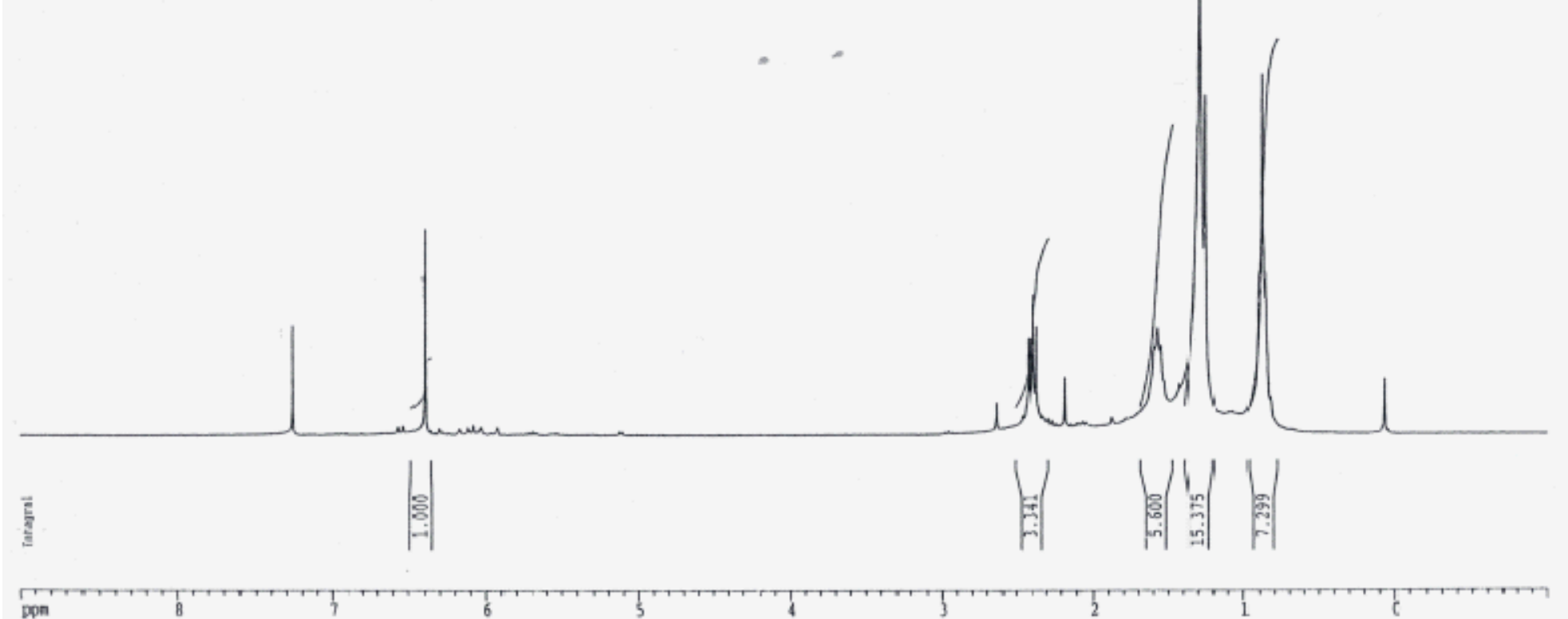


Appendix 2: ${ }^{13} \mathrm{C}$ NMR spectrum of (Z,Z)-7,10-dichlorohexadeca-7,9-diene (3a)

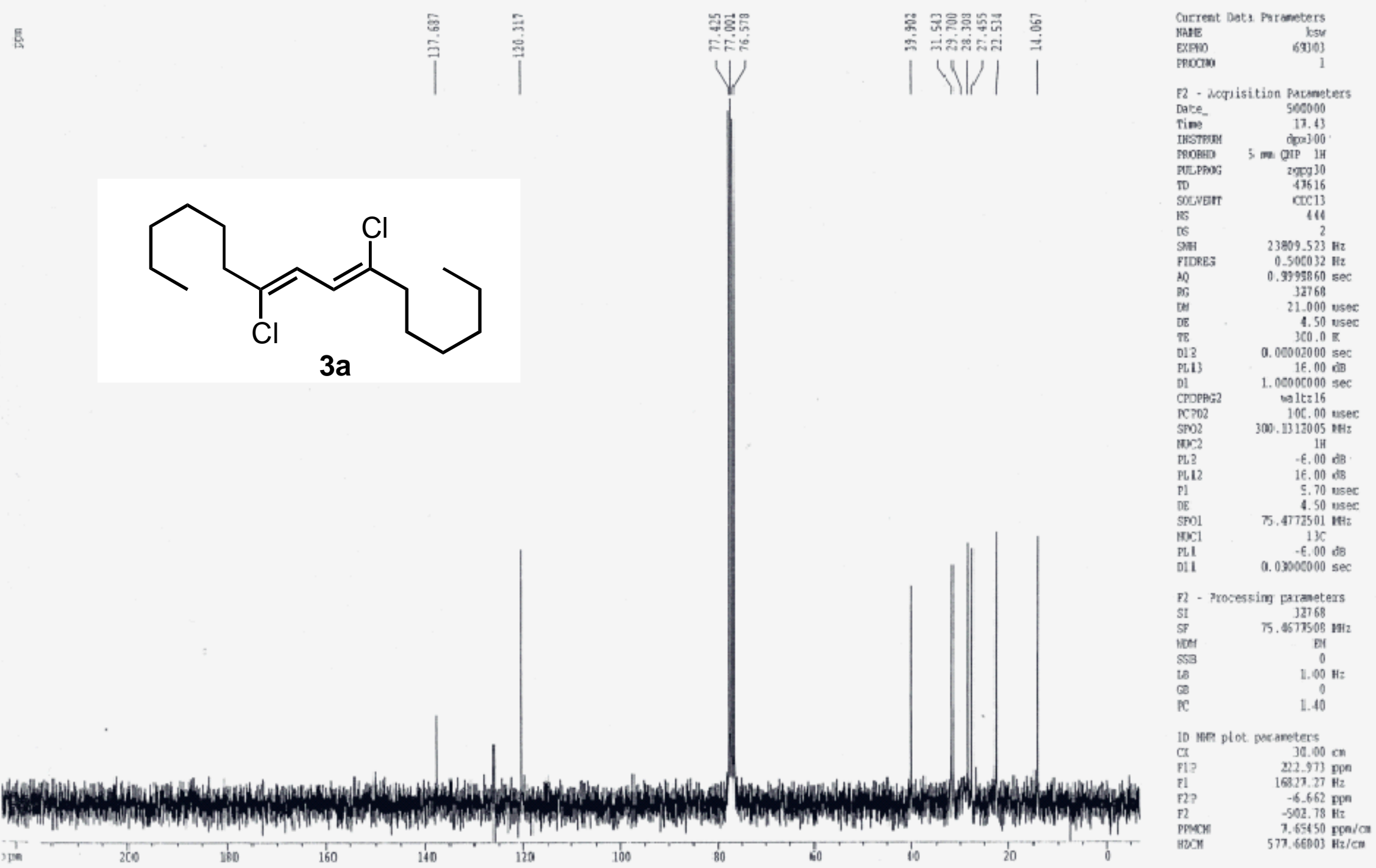


Appendix 3: ${ }^{1} \mathrm{H}$ NMR spectrum of (Z,Z)-1,4,7,10-tetrachloro-4,6-decadiene (3c)

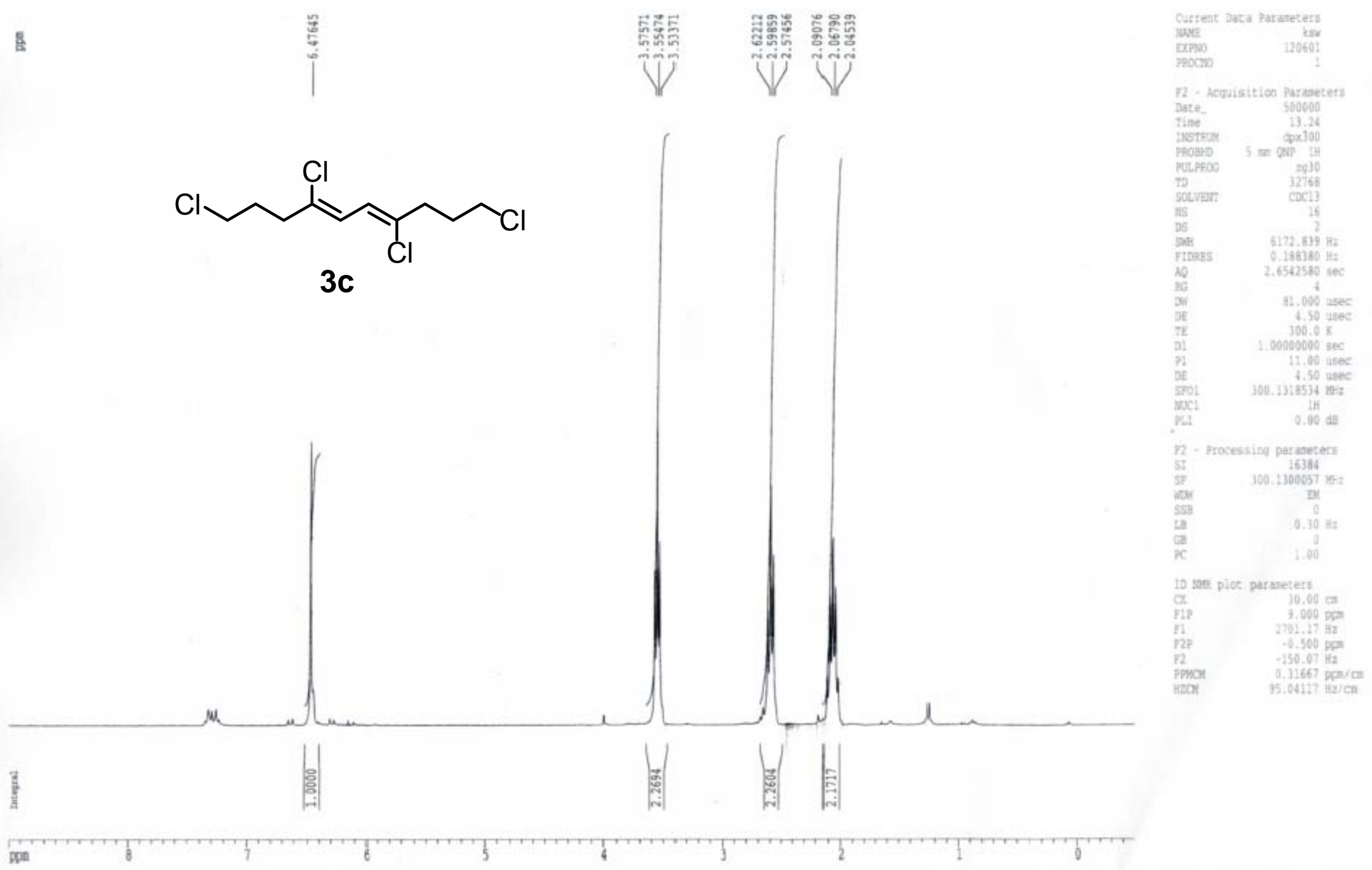


Appendix 4: ${ }^{13} \mathrm{C}$ NMR spectrum of (Z,Z)-1,4,7,10-tetrachloro-4,6-decadiene (3c)

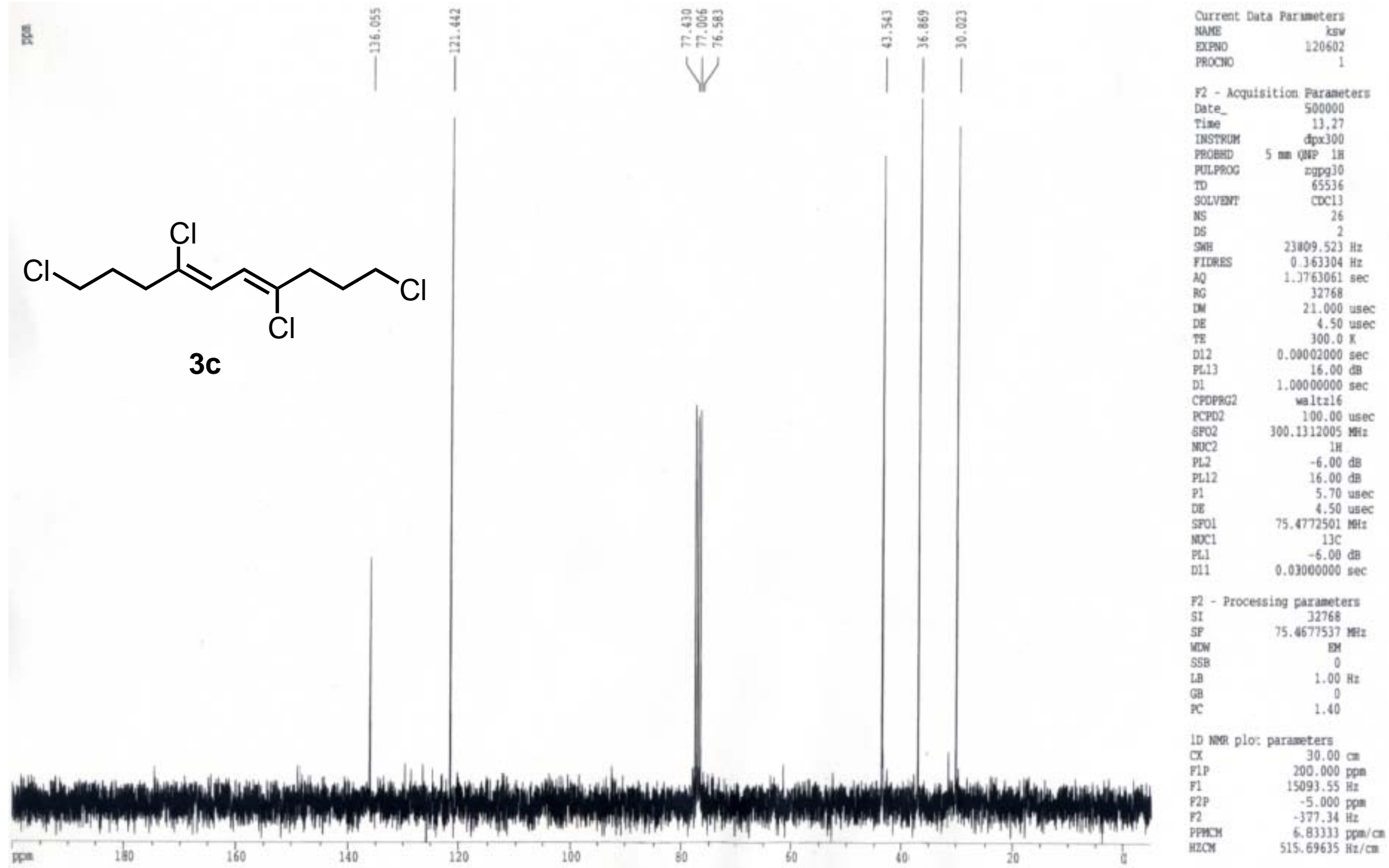


Appendix 5: ${ }^{1} \mathrm{H}$ NMR spectrum of (Z,Z)-5,8-dichlorododeca-5,7-dienodinitrile (3d)

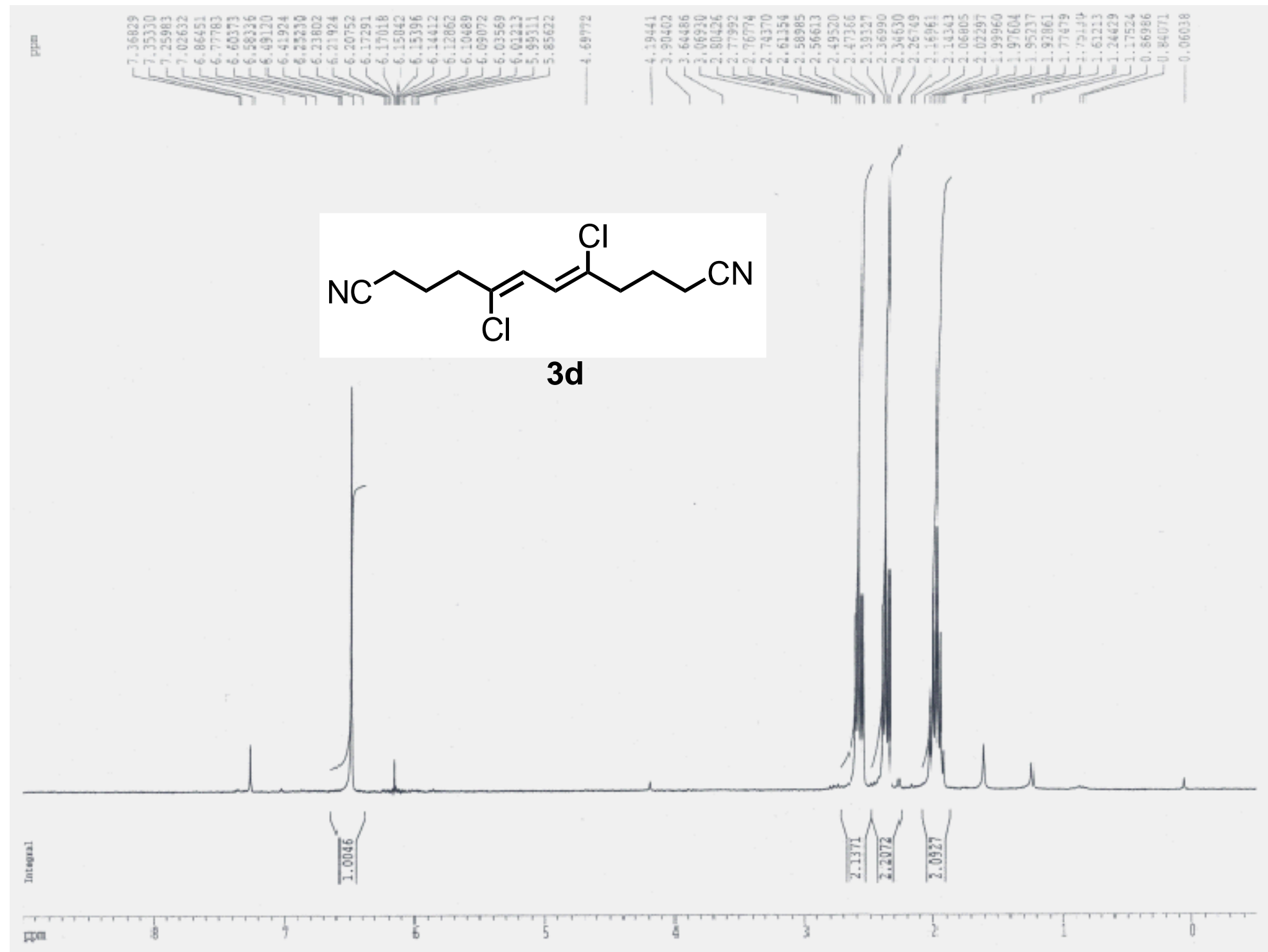


Appendix 6: ${ }^{13} \mathrm{C}$ NMR spectrum of (Z,Z)-5,8-dichlorododeca-5,7-dienodinitrile (3d)

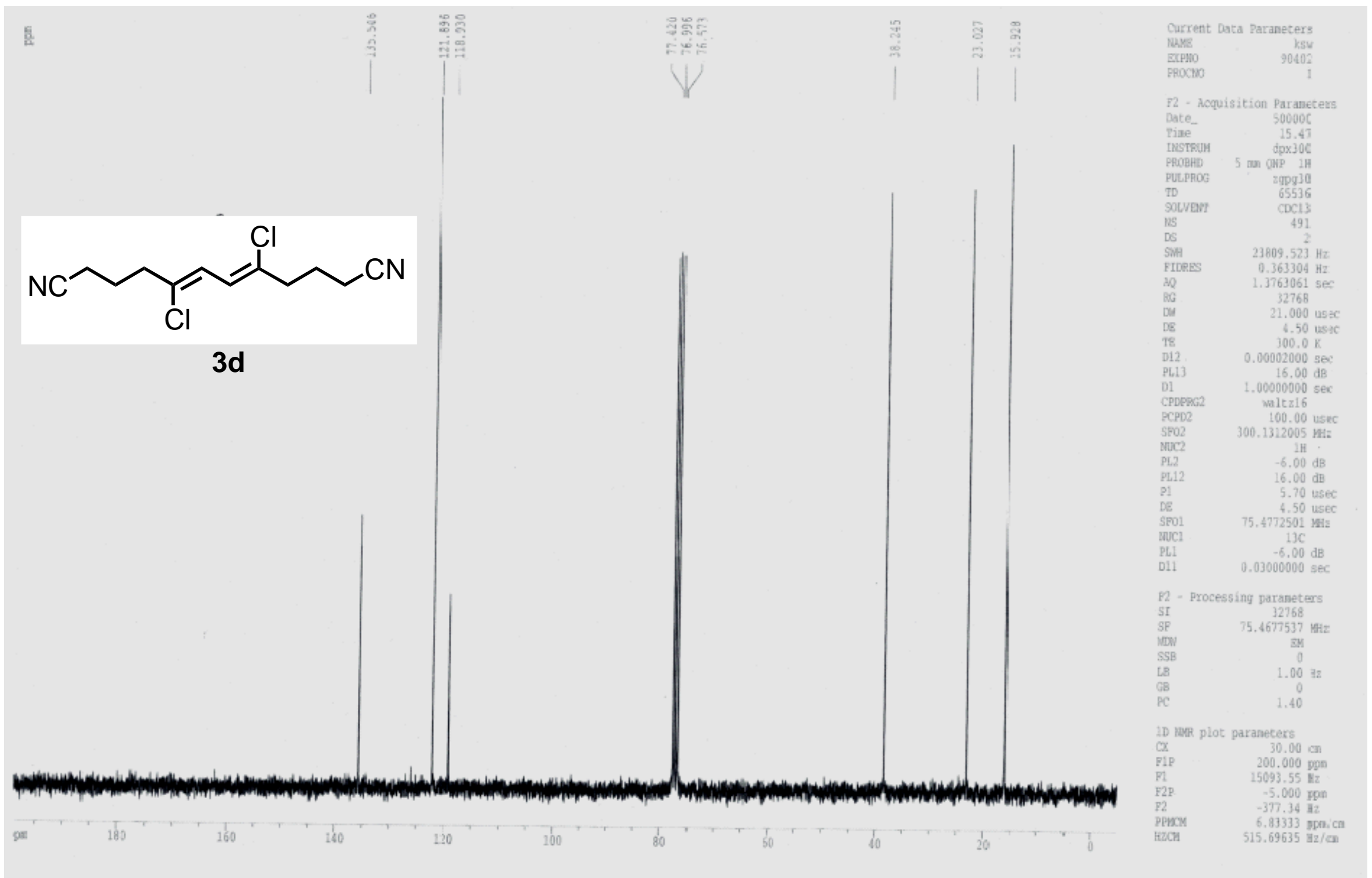


Appendix 7: ${ }^{1} \mathrm{H}$ NMR spectrum of dimethyl (Z,Z)-5,8-dichlorododeca-5,7-dienedioate (3e)

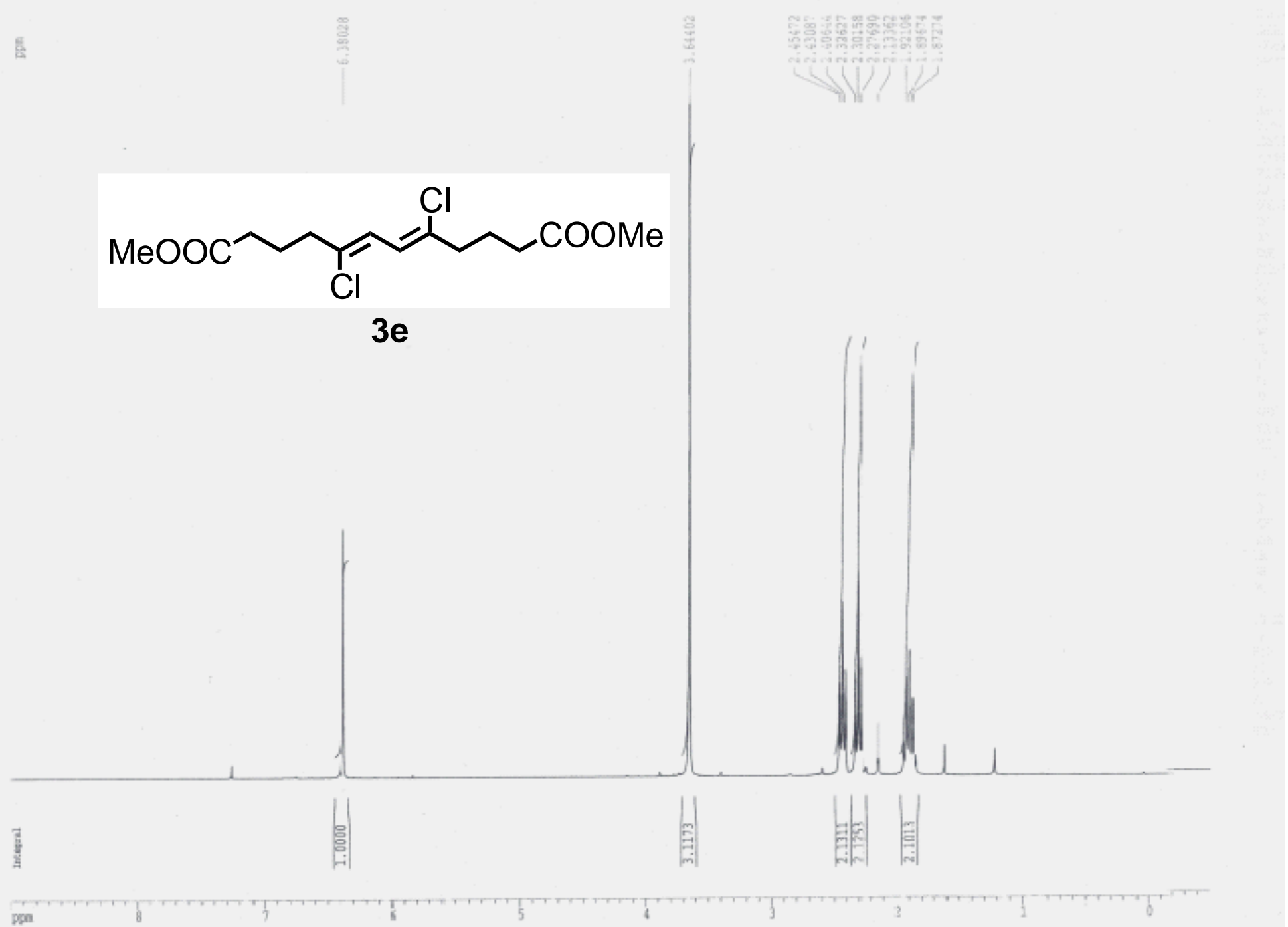


Appendix 8: ${ }^{13} \mathrm{C}$ NMR spectrum of dimethyl (Z,Z)-5,8-dichlorododeca-5,7-dienedioate (3e)

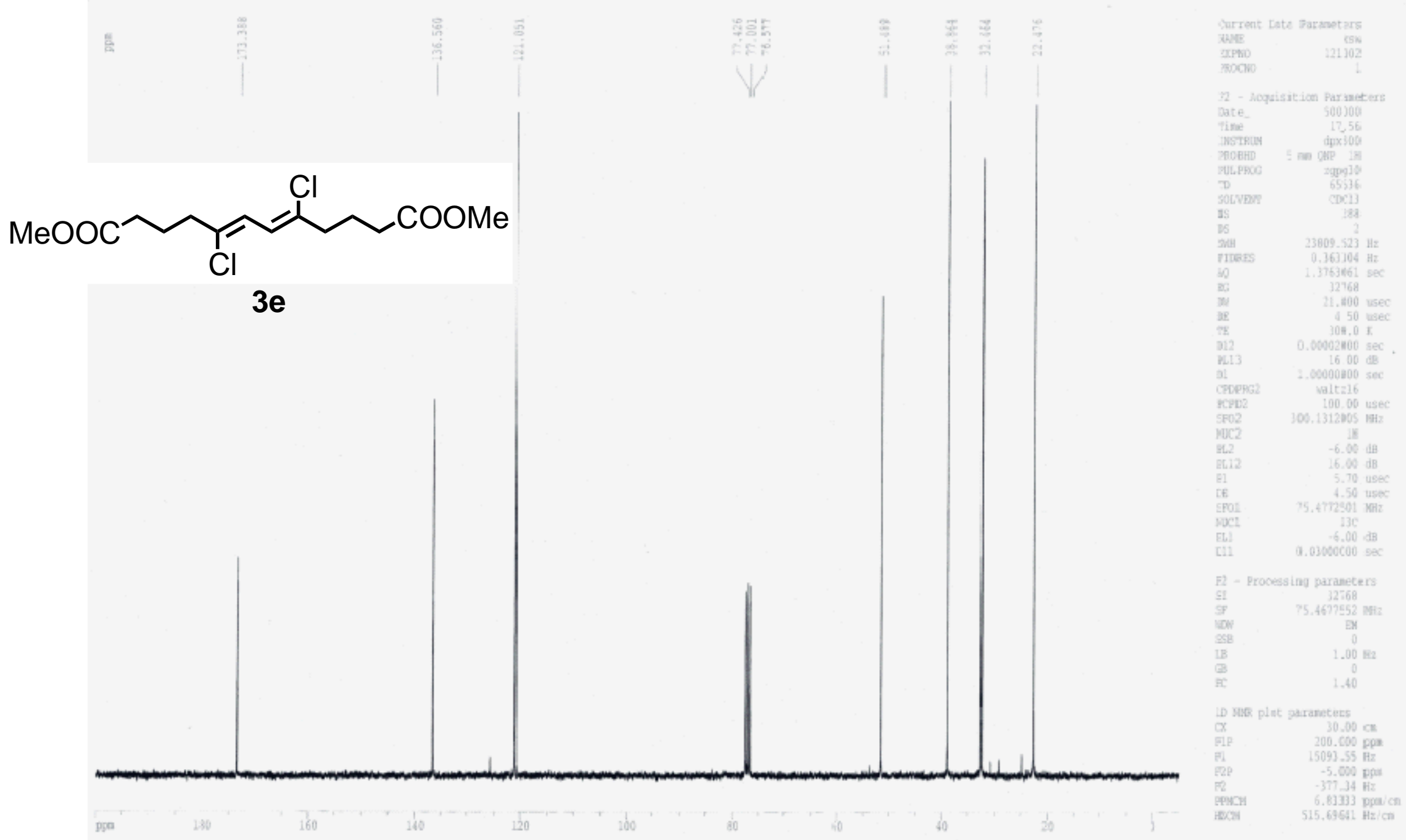


Appendix 9: ${ }^{1} \mathrm{H}$ NMR spectrum of (Z,Z)-1,6-diphenyl-2,5-dichloro-2,4-hexadiene (3f)

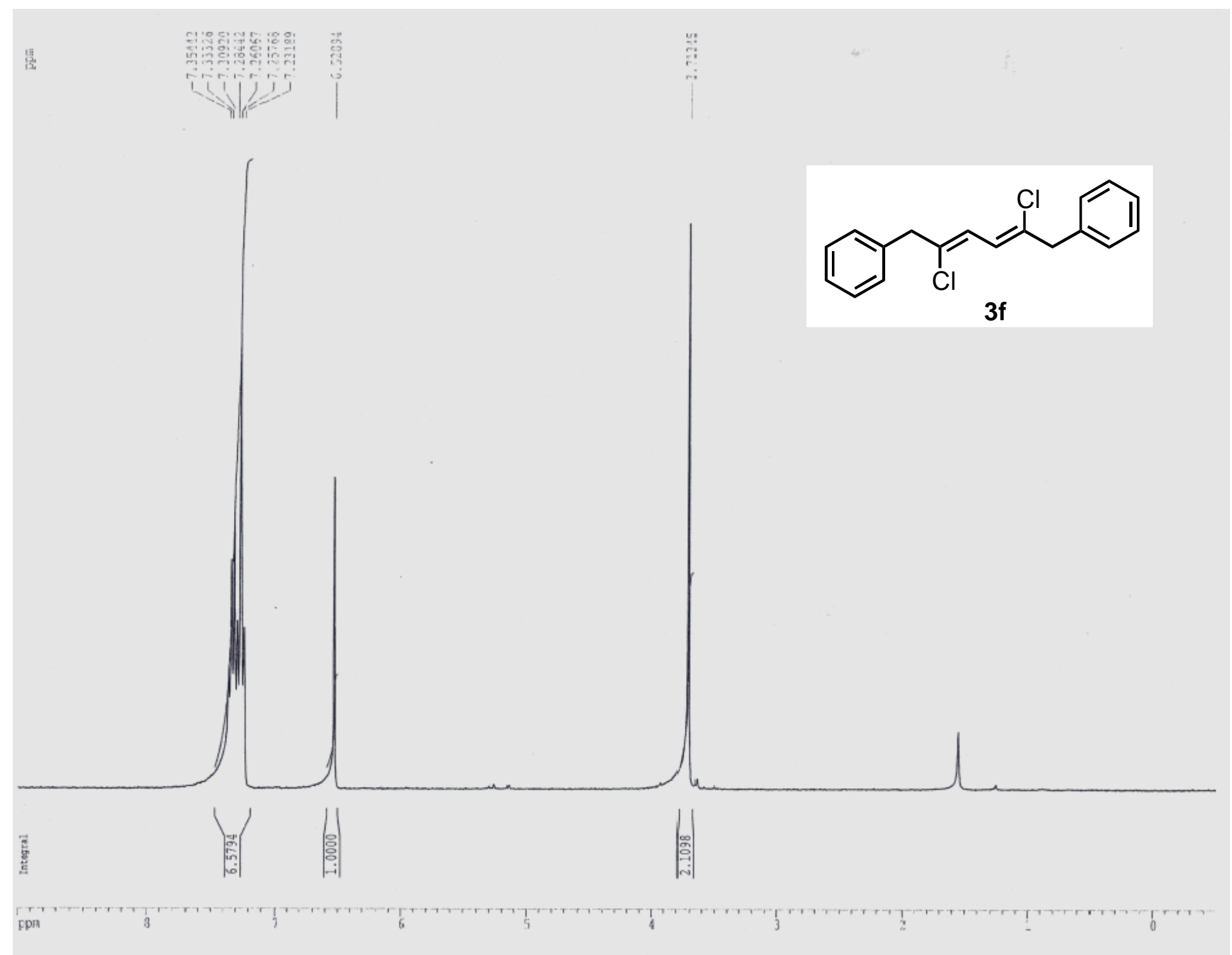


Appendix 10: ${ }^{13} \mathrm{C}$ NMR spectrum of (Z,Z)-1,6-diphenyl-2,5-dichloro-2,4-hexadiene (3f)

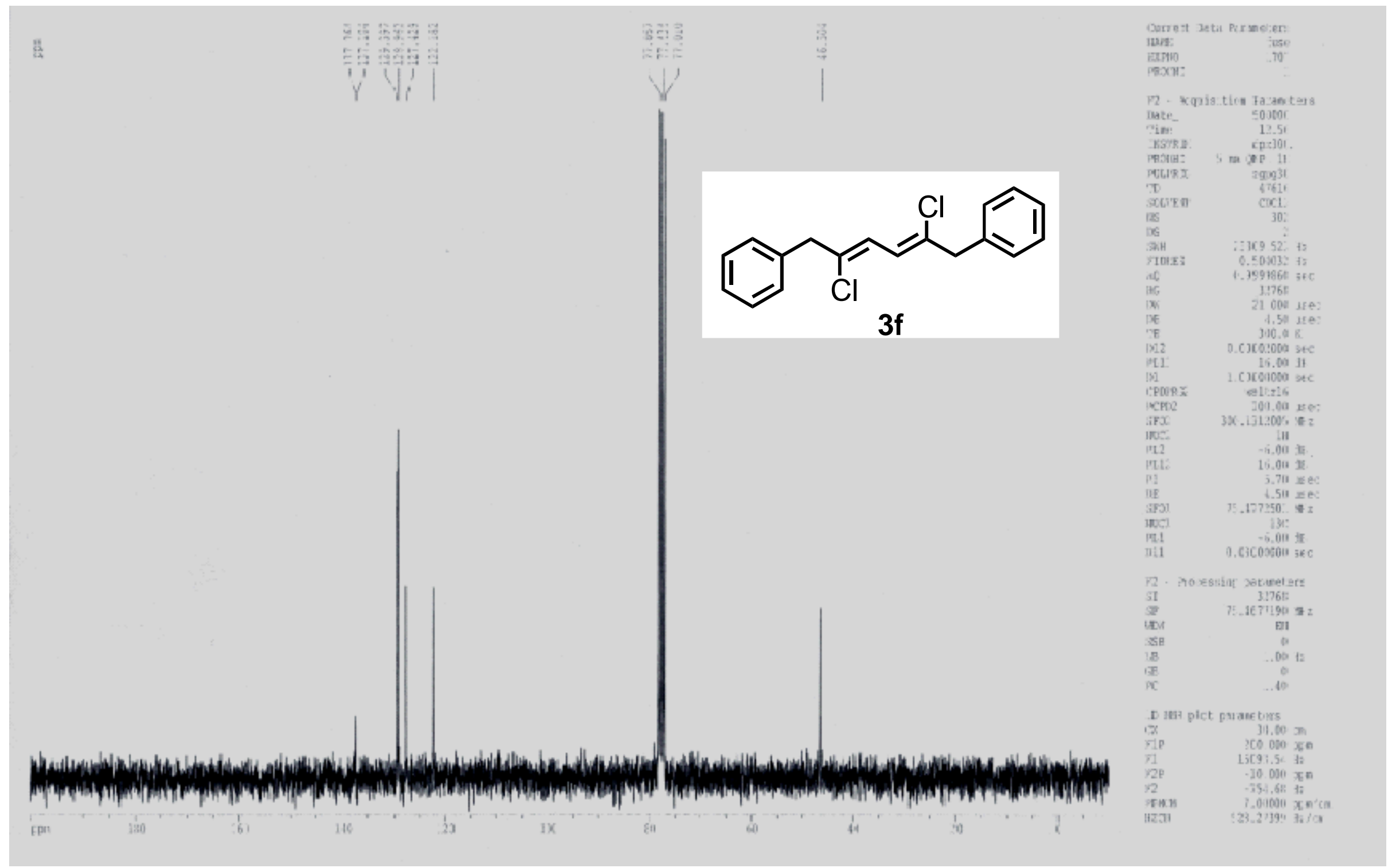


Appendix 11: ${ }^{1} \mathrm{H}$ NMR spectrum of (Z,Z)-1,4-dichloro-1,4-bis(trimethylsilyl)butadiene (3g)

基

$$
\text { i }
$$
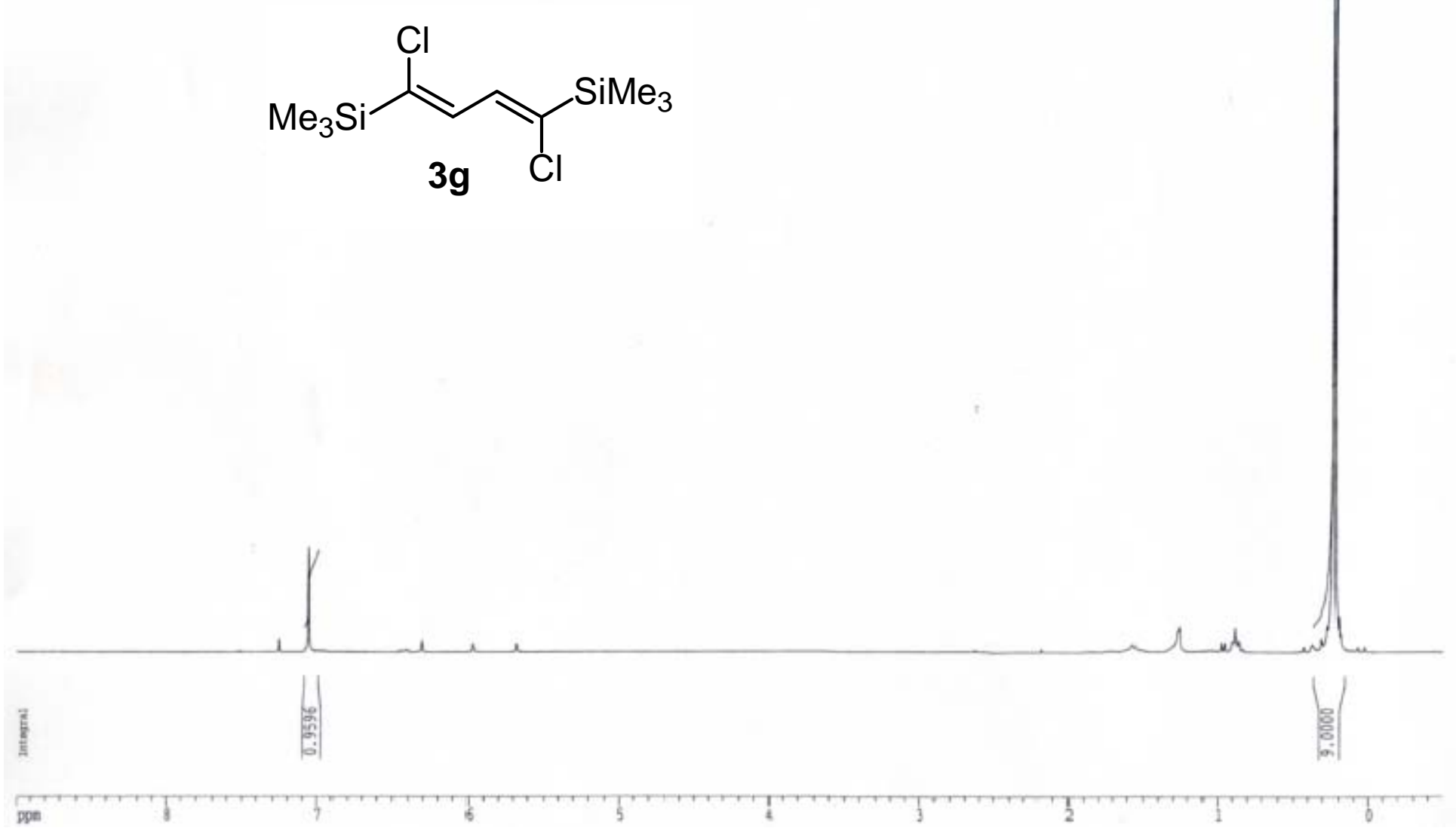

pon

$3 g$

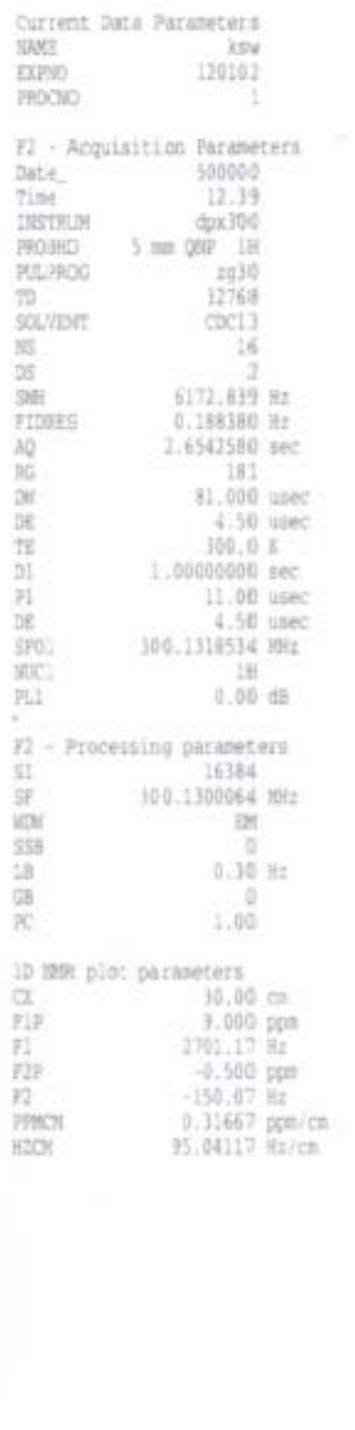


Appendix 12: ${ }^{13} \mathrm{C}$ NMR spectrum of (Z,Z)-1,4-dichloro-1,4-bis(trimethylsilyl)butadiene (3g)

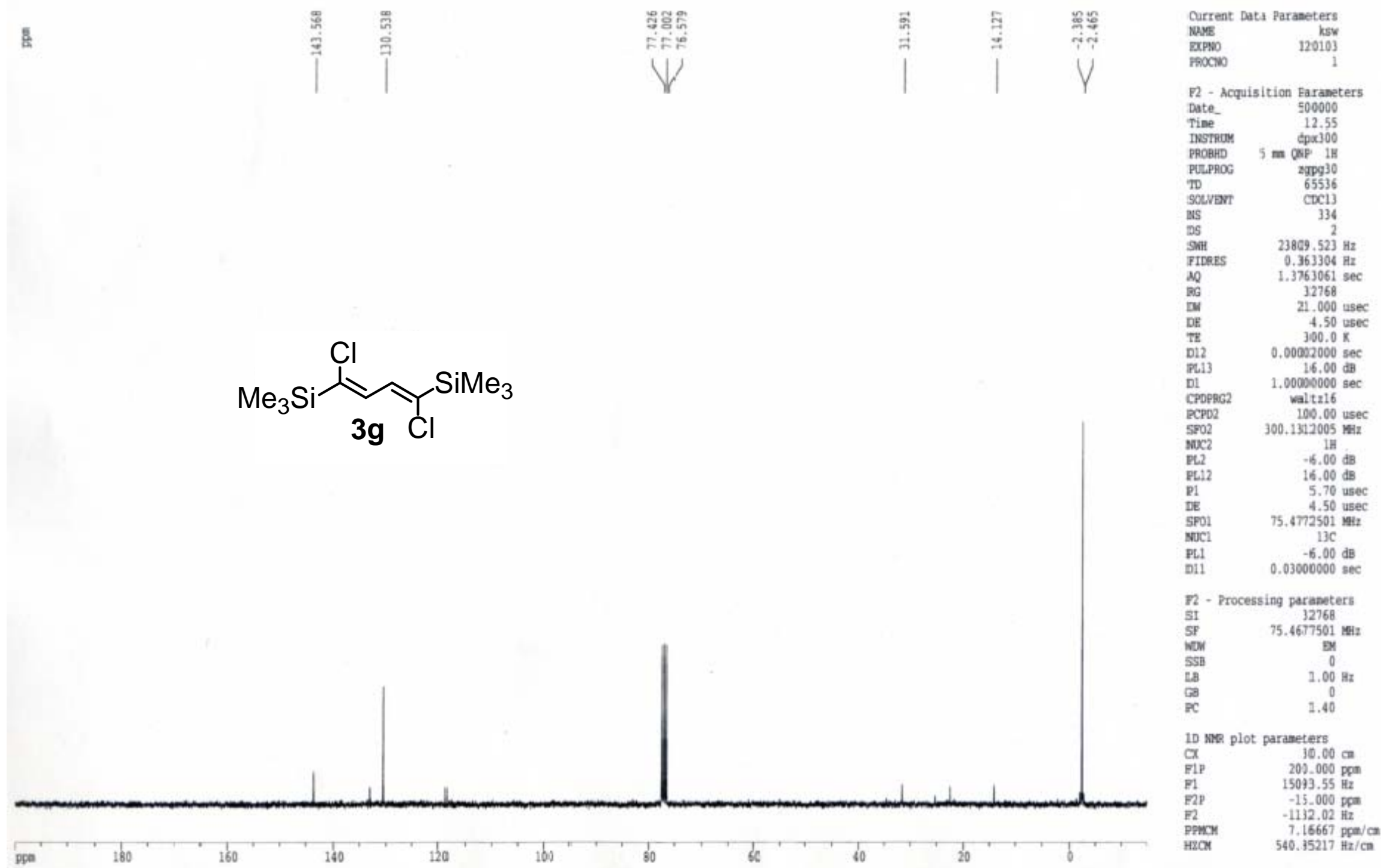


Appendix 13: ${ }^{1} \mathrm{H}$ NMR spectrum of (Z,Z)-1,4-dichloro-1,4-bis(1-cyclohexenyl)butadiene (3h)

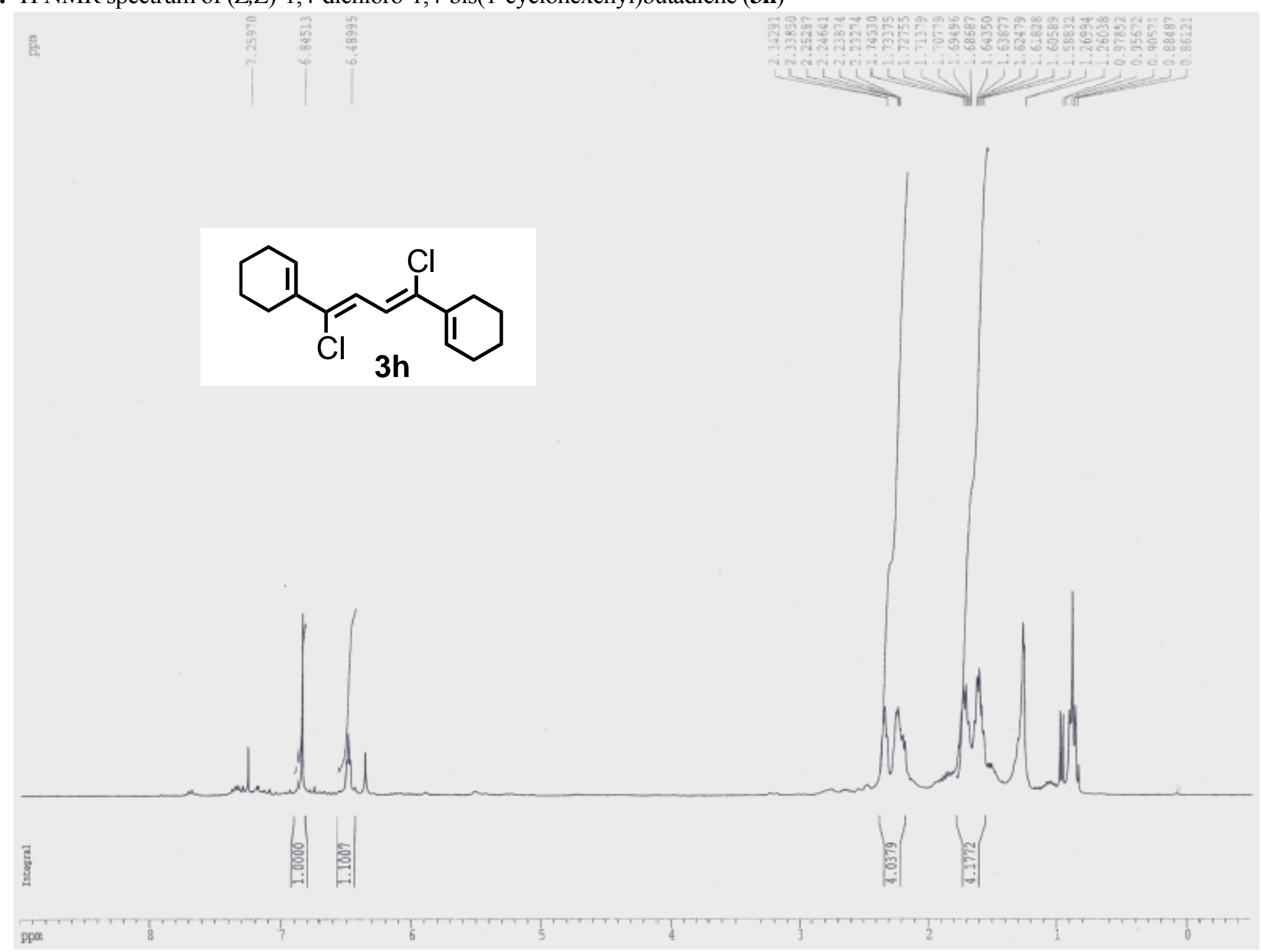


Appendix 14: ${ }^{13} \mathrm{C}$ NMR spectrum of (Z,Z)-1,4-dichloro-1,4-bis(1-cyclohexenyl)butadiene (3h)

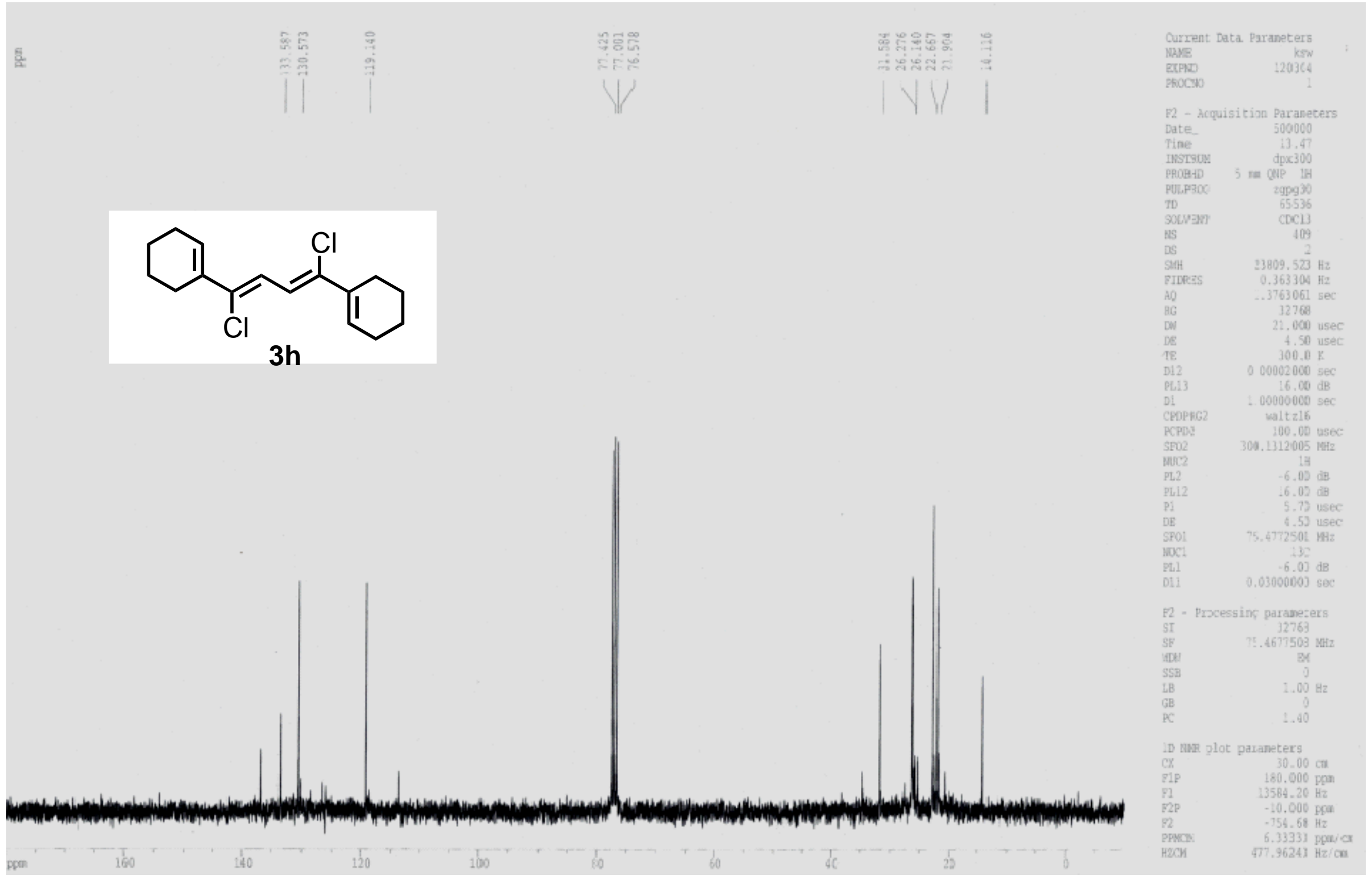


Appendix 15: ${ }^{1} \mathrm{H}$ NMR spectrum of (Z,Z)-1,4-dichloro-1,4-di(4-methoxyphenyl)-1,3-butadiene (3j)

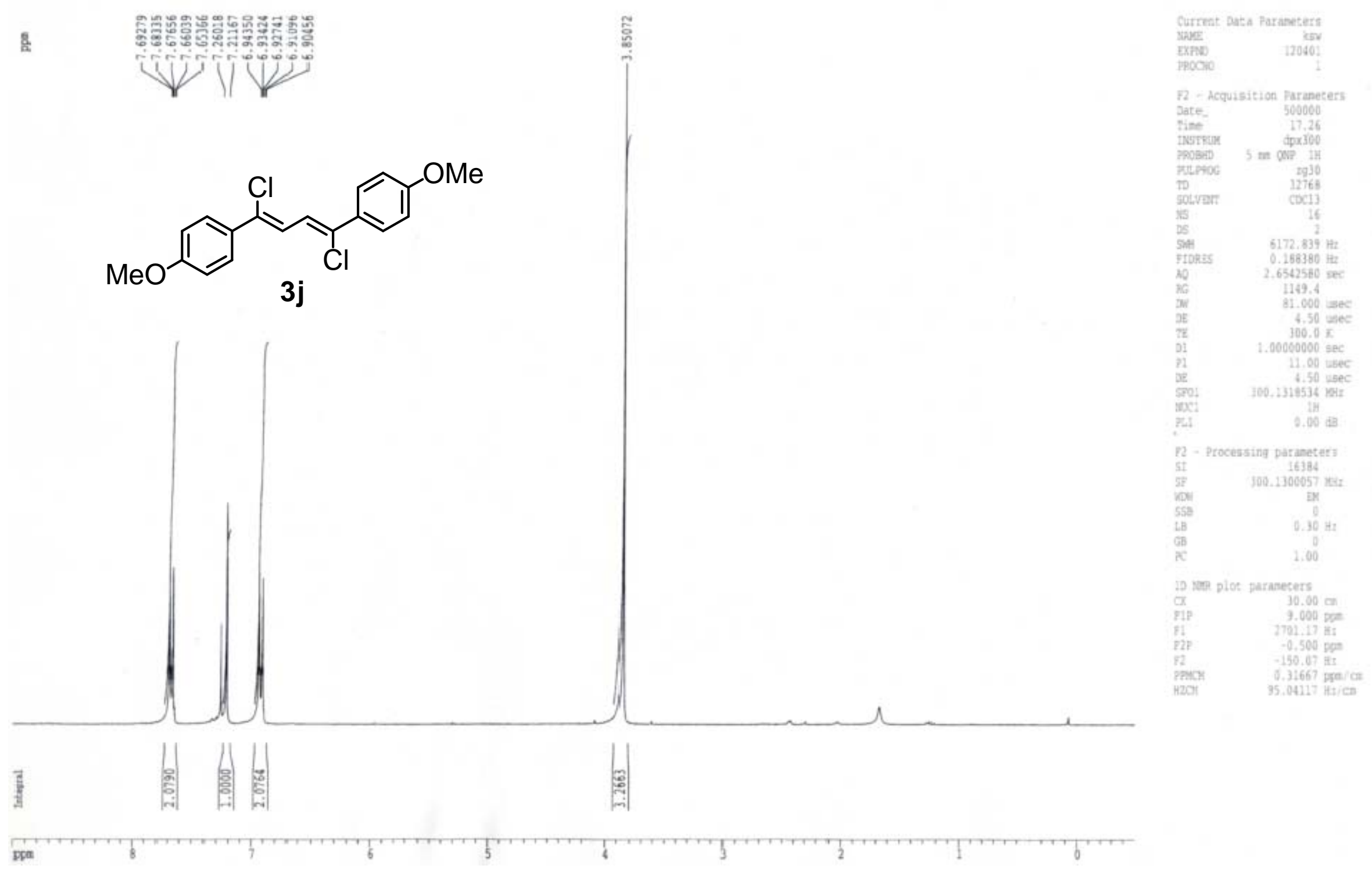


Appendix 16: ${ }^{13} \mathrm{C}$ NMR spectrum of (Z,Z)-1,4-dichloro-1,4-di(4-methoxyphenyl)-1,3-butadiene (3j)

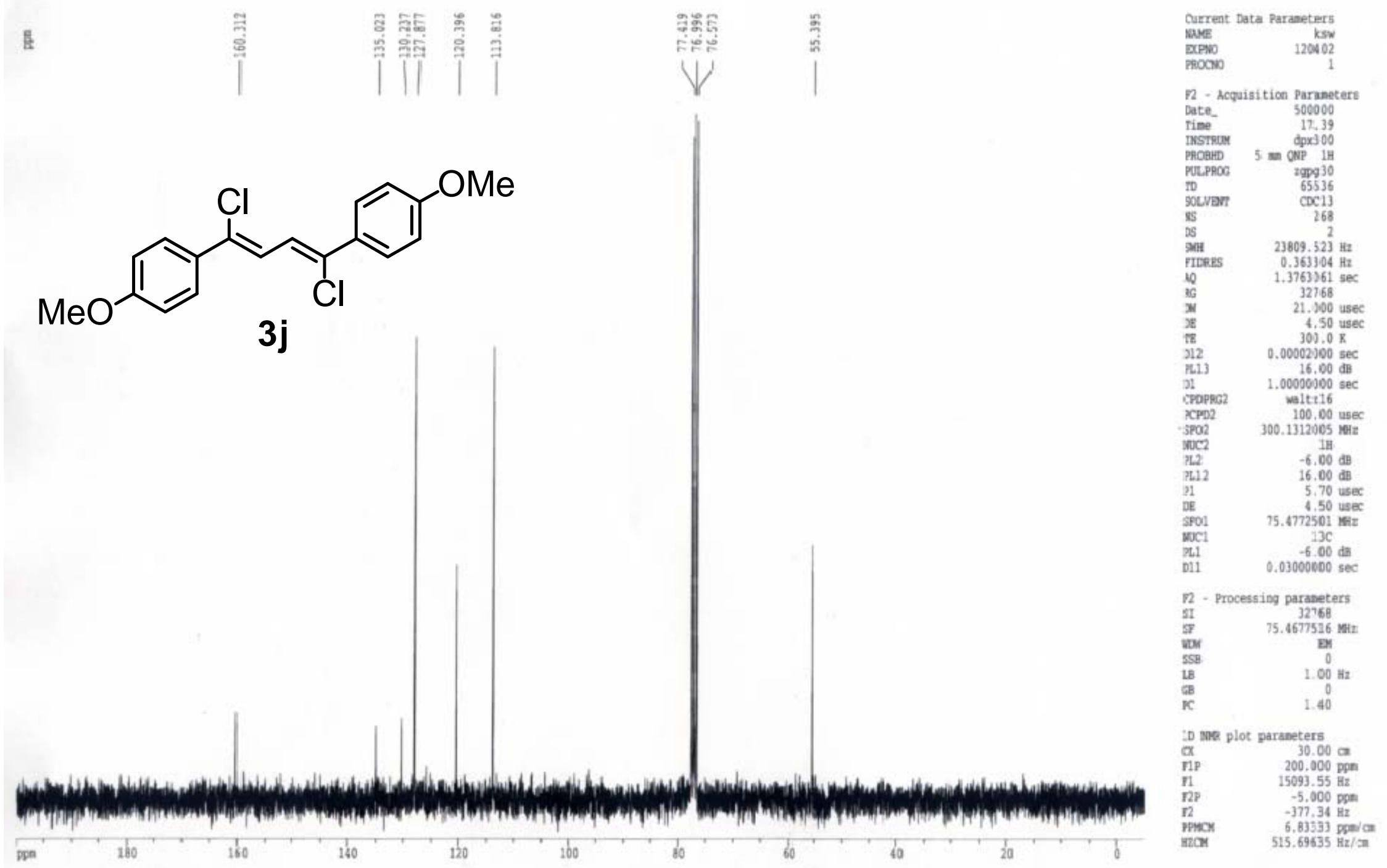


Appendix 17: ${ }^{1} \mathrm{H}$ NMR spectrum of (Z,Z)-1,4-dichloro-1,4-di(4-fluorophenyl)-1,3-butadiene (3l)

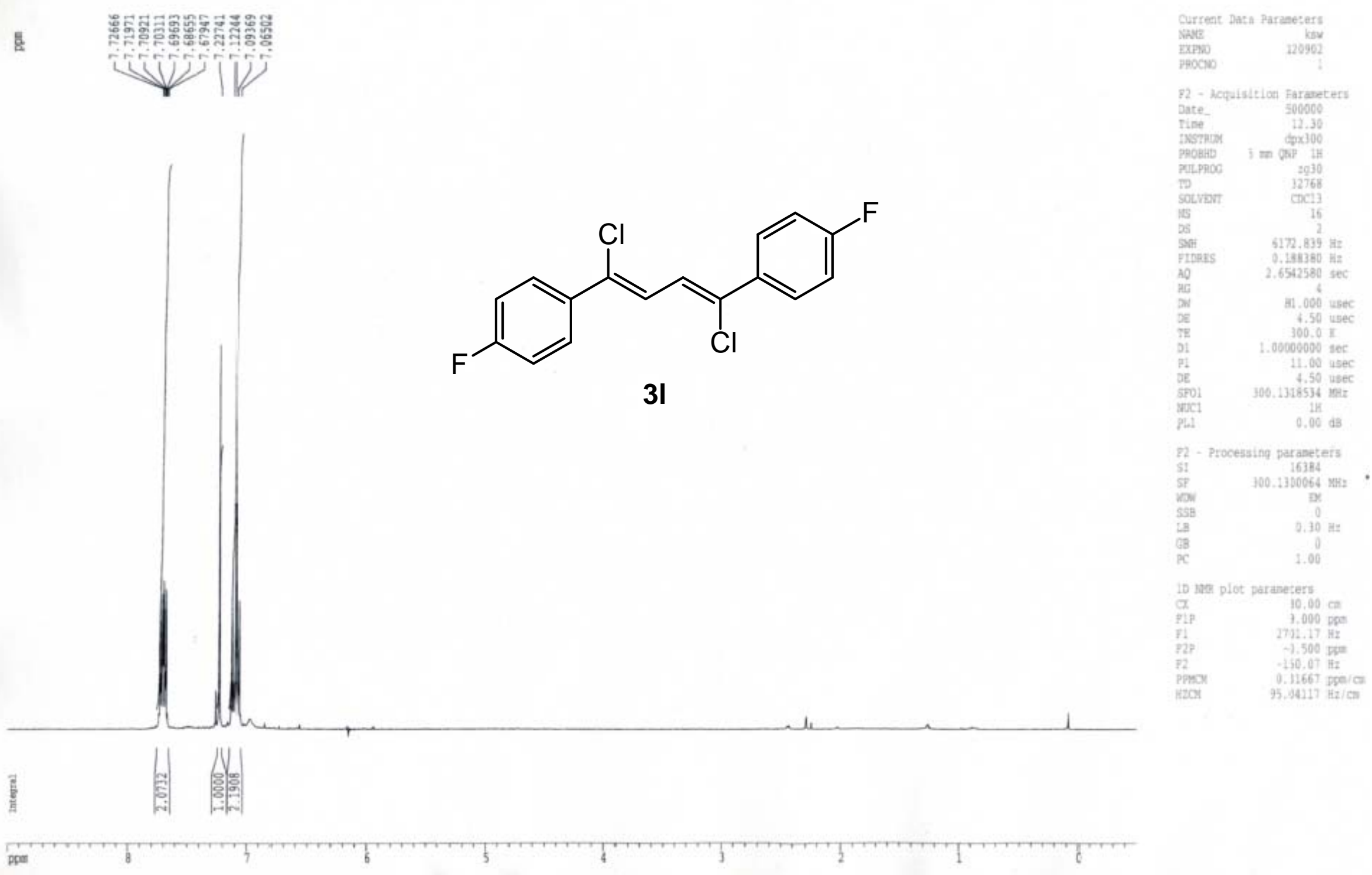


Appendix 18: ${ }^{13} \mathrm{C}$ NMR spectrum of (Z,Z)-1,4-dichloro-1,4-di(4-fluorophenyl)-1,3-butadiene (3l)

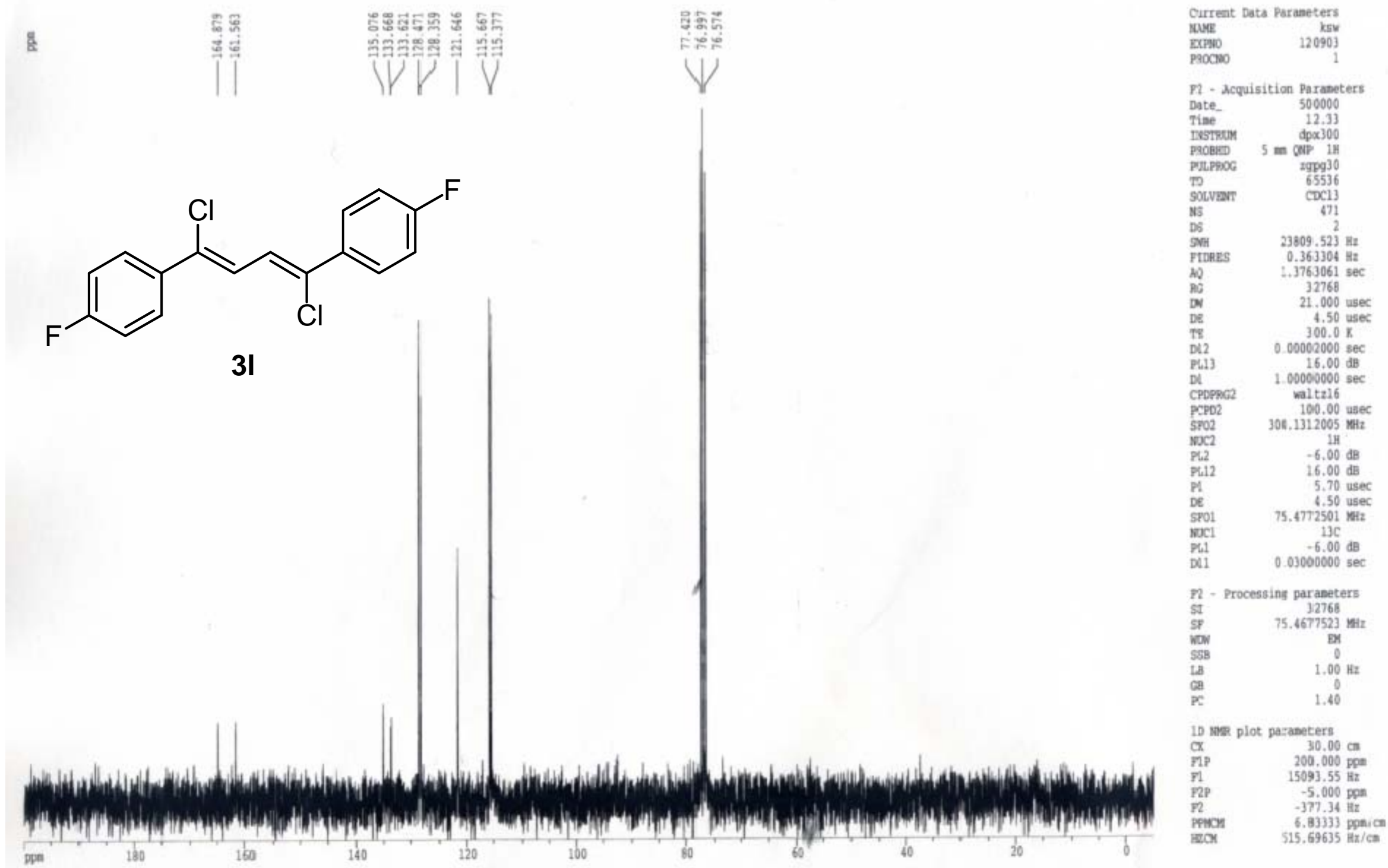


Appendix 19: ${ }^{1} \mathrm{H}$ NMR spectrum of (Z,Z)-1,4-dichloro-1,4-di(2-thienyl)-1,3-butadiene (3m)
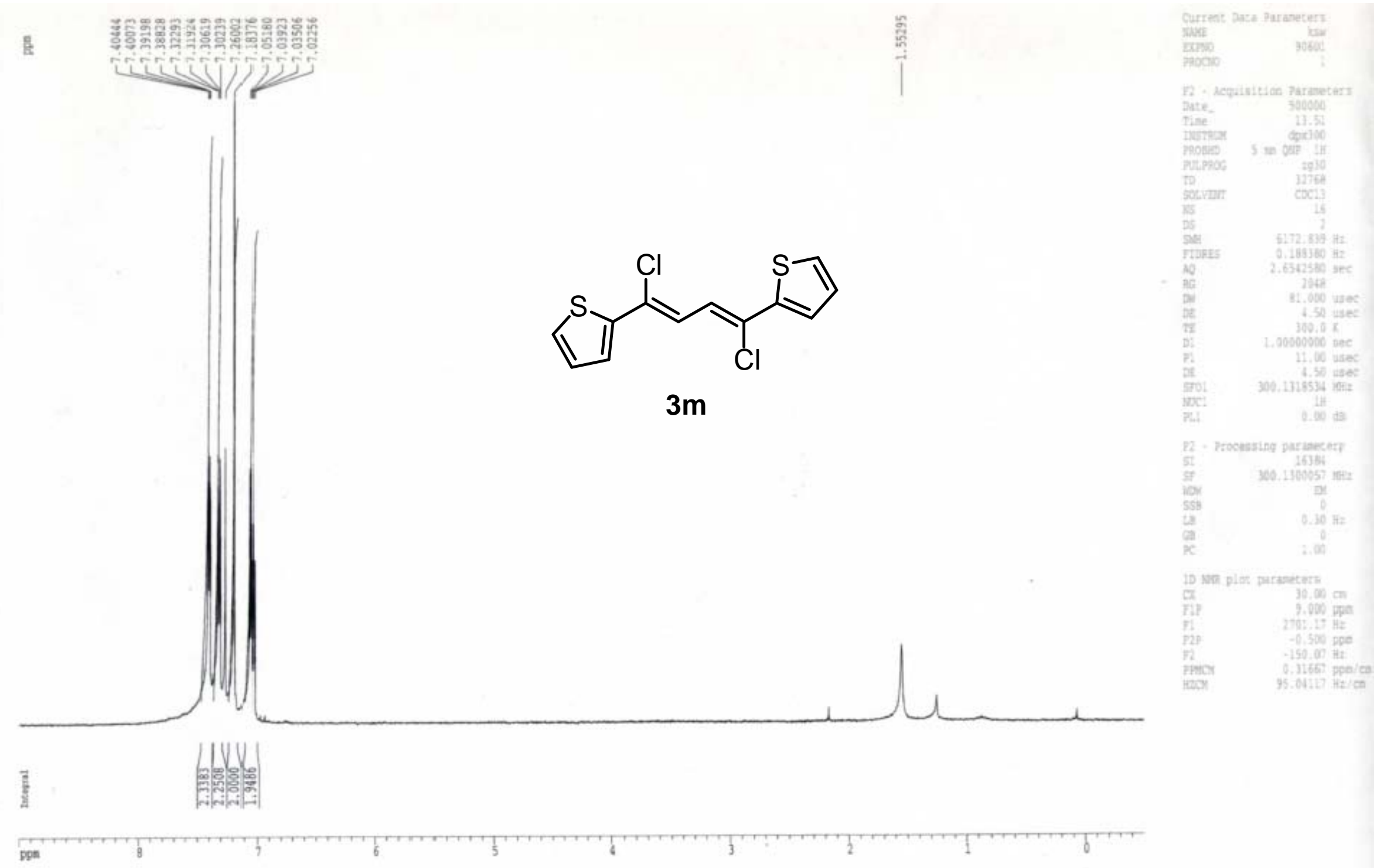
Appendix 20: ${ }^{13} \mathrm{C}$ NMR spectrum of (Z,Z)-1,4-dichloro-1,4-di(2-thienyl)-1,3-butadiene (3m)

g<smiles>Cl/C(=C\C=C(/Cl)c1cccs1)c1cccs1</smiles>

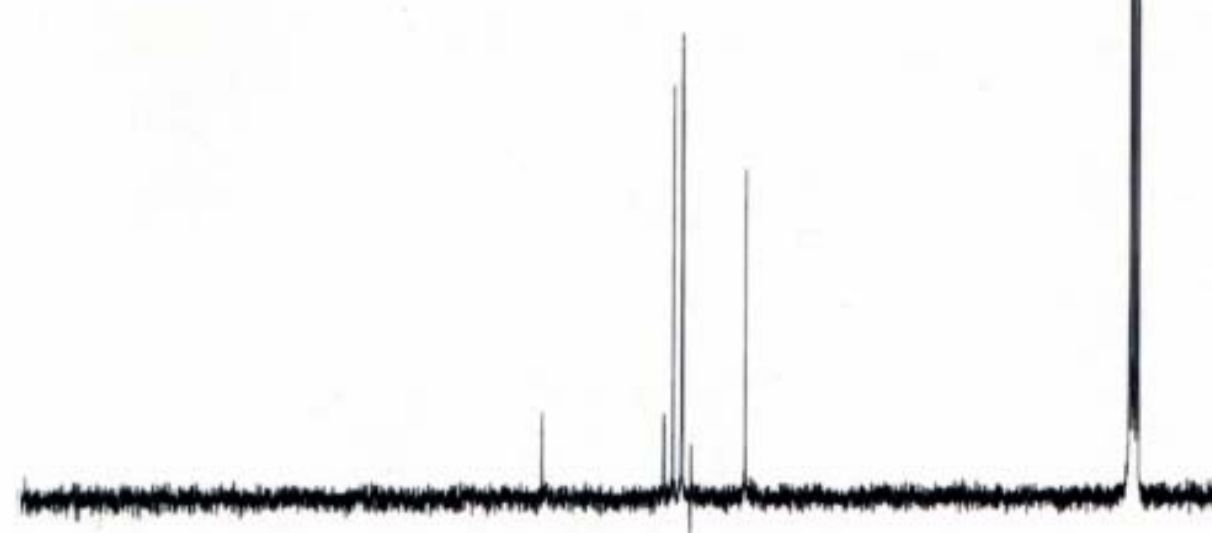

pan
$3 m$

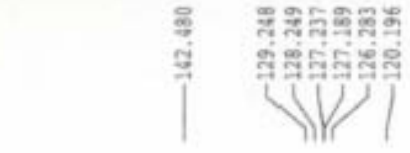

3

ite

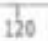

100

bo

60

is 10

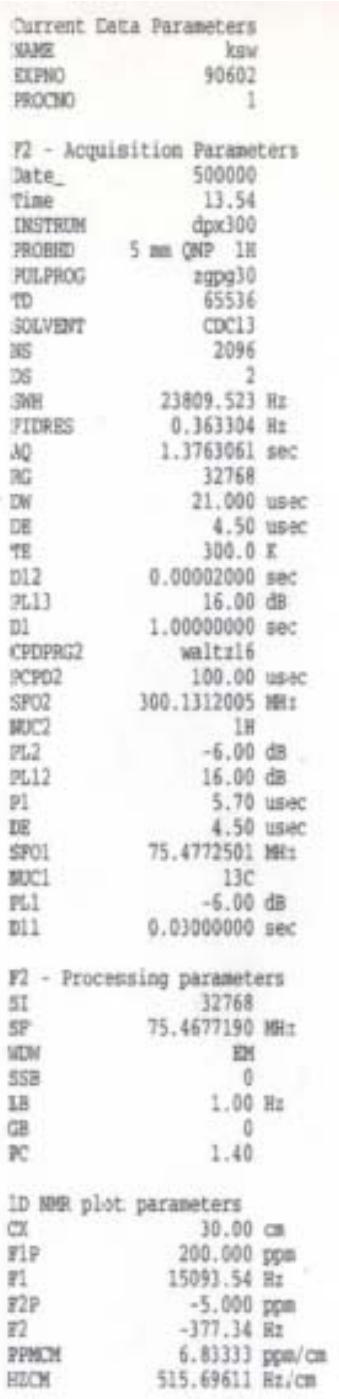


Appendix 21: ${ }^{1} \mathrm{H}$ NMR spectrum of (Z,Z)-3,6-dichloro-2,2,7,7-tetramethyl-3,5-octadiene (3b)
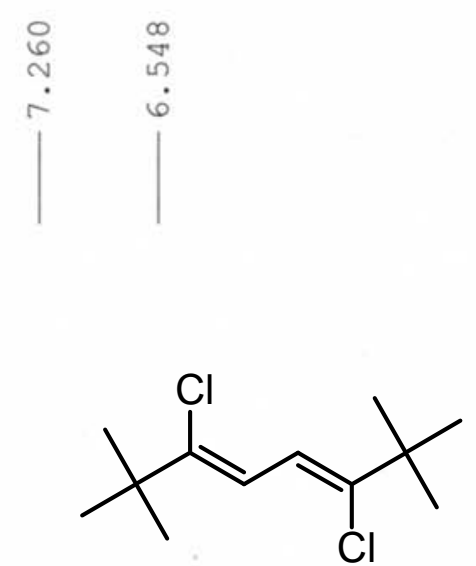

$3 b$
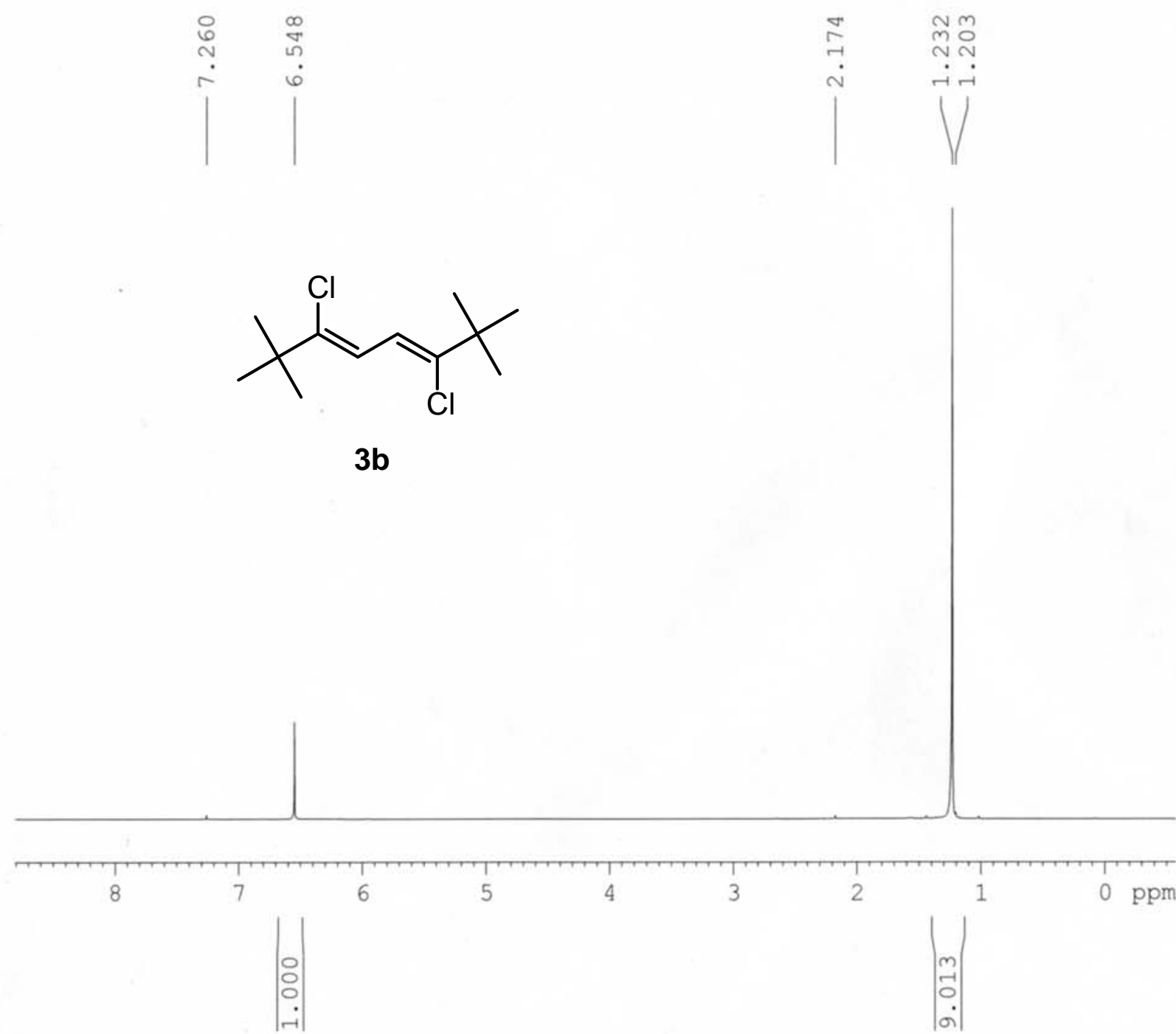

Current Data Parameters

$\begin{array}{lr}\text { NAME } & \text { kSW } \\ \text { EXPNO } & 120501\end{array}$

F2 - Acquisition Parameters

Date_ 500000

Time 18.46

INSTRUM $\quad \mathrm{dp} \times 300$

$5 \mathrm{~mm}$ QNP 1 PROBHD

TD

SOLVEN

DS

TIDRES $\quad 0.188380 \mathrm{~Hz}$

DW $\quad 81.000$ usec

DE $\quad 4.50$ usec

$\begin{array}{ll}\text { TE } & 300.0 \mathrm{~K} \\ \mathrm{D} 1 & 1.00000000 \mathrm{sec}\end{array}$

$\begin{array}{lr}\text { D1 } & 1.00000000 \mathrm{sec} \\ \text { P1 } & 11.00 \mathrm{usec}\end{array}$

DE $\quad 4.50$ usec

SFO1 $\quad 300.1318534 \mathrm{MHz}$

NUC1 1 H

F2 - Processing parameters
SI
16384

$\begin{array}{lc}\text { SF } & 300.1300060 \mathrm{MHz} \\ \text { WDW } & \text { EM }\end{array}$

LB

PC

$0.30 \mathrm{~Hz}$

1.00 
Appendix 22: ${ }^{13} \mathrm{C}$ NMR spectrum of (Z,Z)-3,6-dichloro-2,2,7,7-tetramethyl-3,5-octadiene (3b)

s

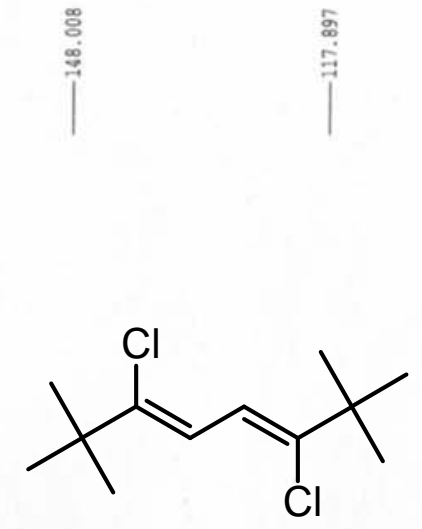

3b

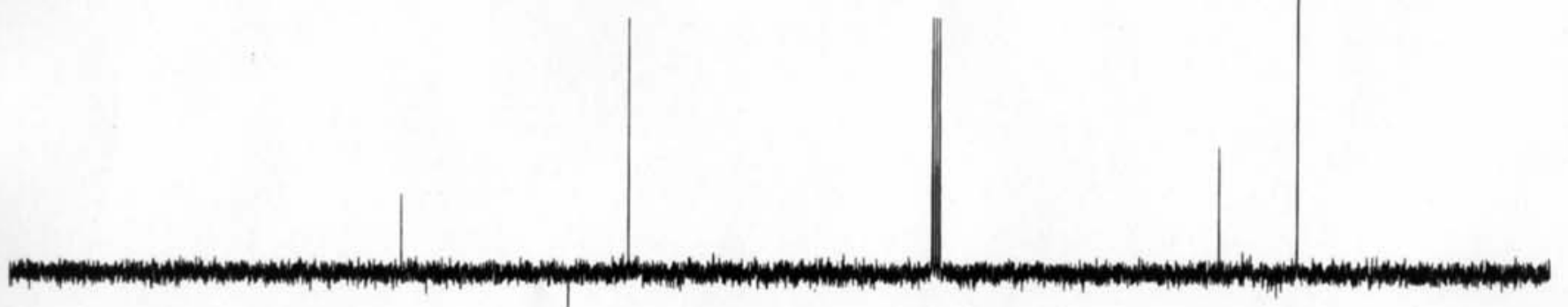

ppo

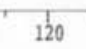

120

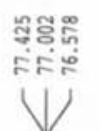

80

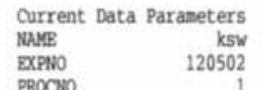

P2 - Acquisition Parameters

$\begin{array}{lr}\text { Date- } & 500000 \\ \text { Time } & 18.49\end{array}$

Time
INSTRUM
PROBHD
PUP $5=$ aWP 1800

PGpg 30
PULPROG
TD

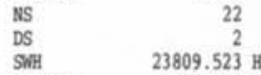

PIDRES $\quad 0.363304 \mathrm{~Hz}$

$\begin{array}{ll}\text { Al } & 1.3763061 \mathrm{sec} \\ \mathrm{RG} & 32768\end{array}$

$\begin{array}{lr}21.000 \text { usec } \\ \text { DE } & 4.50 \text { usec }\end{array}$

$\begin{array}{ll}\text { IR } & 300.0 \mathrm{~K} \\ \mathrm{D} 12 & 0.00002000 \mathrm{sec}\end{array}$

$0.00002000 \mathrm{sec}$
$\mathrm{PL13}$

$\begin{array}{ll}\mathrm{Di} & 1.00000000 \mathrm{sec} \\ \text { CPDPRG2 } & \text { waltz216 }\end{array}$

$\begin{array}{ll}\text { PCPD2 } & 100.00 \mathrm{usec} \\ \text { SPO2 } & 300.1312005 \mathrm{sti2}\end{array}$

$\begin{array}{ll}\mathrm{NOCC} & 1 \mathrm{~B} \\ \mathrm{PLL} & -6.00 \mathrm{~dB} \\ \mathrm{PL12} & 16.00 \mathrm{~dB}\end{array}$

$\begin{array}{lr}\text { PL12 } & 16.000 \mathrm{~dB} \\ \mathrm{P} & 5.70 \mathrm{usec} \\ \mathrm{DE} & 4.50 \mathrm{usec} \\ \mathrm{DE} & 75.4772503 \mathrm{~s}\end{array}$

SFO1 $\quad 75.4772501 \mathrm{MHz}$

$\begin{array}{ll}\text { NOC1 } & 13 \mathrm{C} \\ \text { PL1 } & -6.00 \mathrm{~dB}\end{array}$

72 - processing parameters

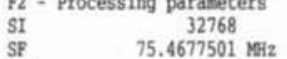

WF $\quad 75.4677501$ EN

$\begin{array}{ll}\text { SSB } & 0 \\ \text { LB } & 1.00 \mathrm{~Hz} \\ \text { GB } & 0 \\ \text { PC } & 1.40\end{array}$

10 me plot parameters

$\begin{array}{lr}\text { ID MMR plot parameters } \\ \text { CX } \\ \text { P1P } & 30.00 \mathrm{~cm} \\ \text { P1 } & 200.000 \mathrm{ppm} \\ \mathrm{PI} & 15093.55 \mathrm{~Hz}\end{array}$

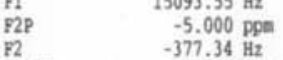

$\begin{array}{lr}\text { PPMCM } & 6.83333 \mathrm{ppm} / \mathrm{cm} \\ \text { H2OM } & 515.69635 \mathrm{~Hz} / \mathrm{Cm}\end{array}$ 
Appendix 23: ${ }^{1} \mathrm{H}$ NMR spectrum of (Z,Z)-1,4-dichloro-1,4-diphenyl-1,3-butadiene (3i)
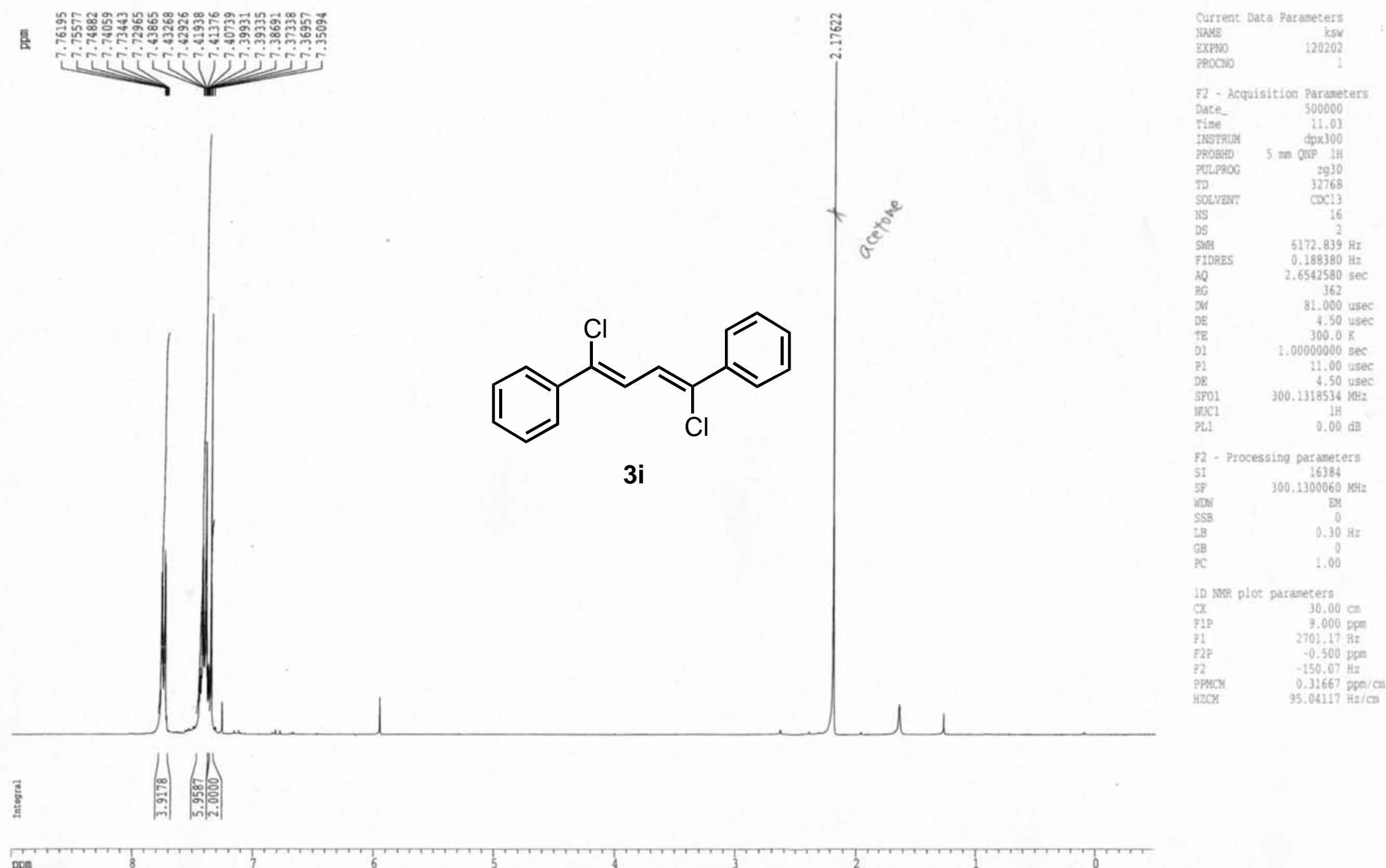
Appendix 24: ${ }^{13} \mathrm{C}$ NMR spectrum of (Z,Z)-1,4-dichloro-1,4-diphenyl-1,3-butadiene (3i)

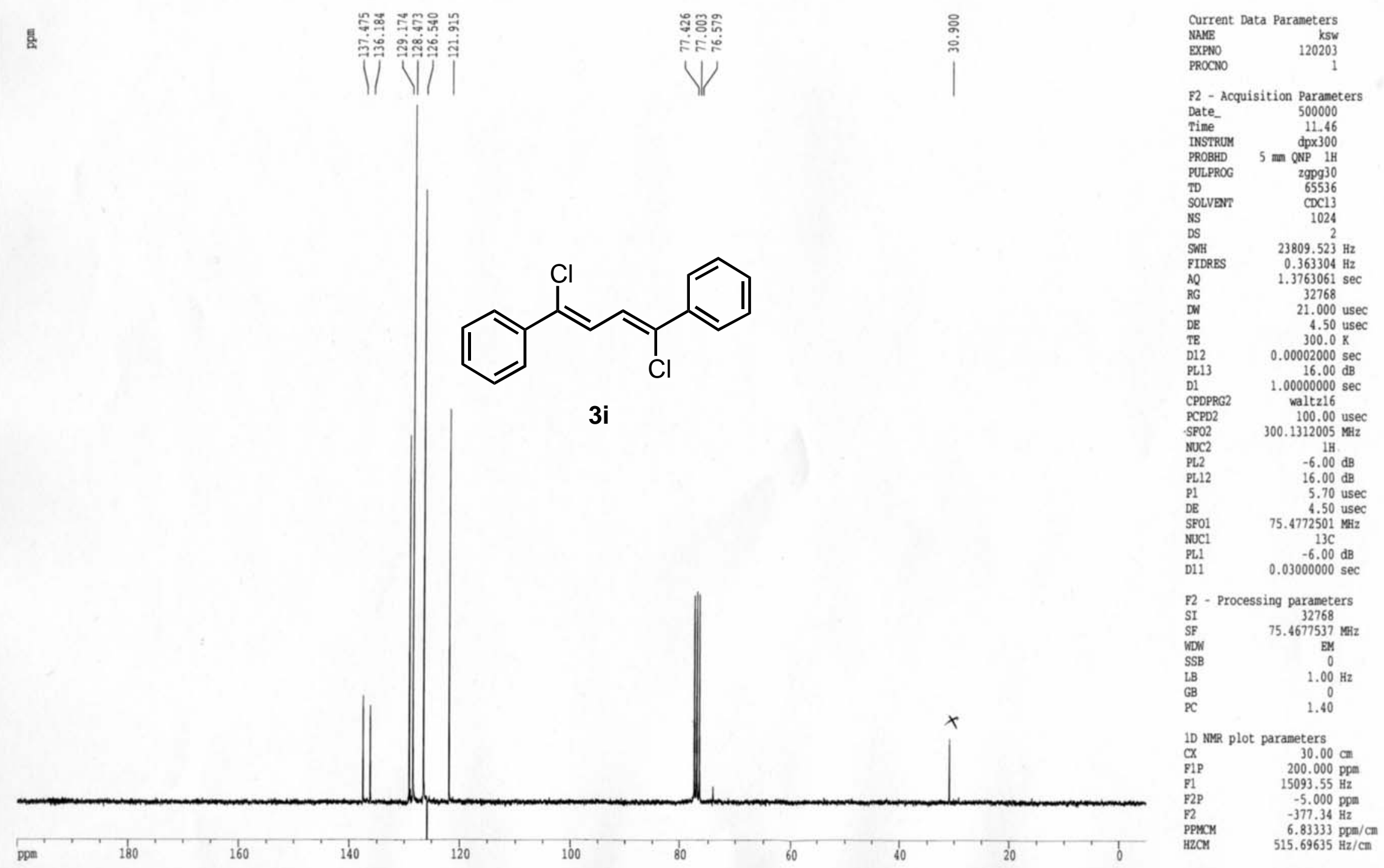


Appendix 25: ${ }^{1}$ H NMR spectrum of (Z,Z)-1,4-dichloro-1,4-di(p-tolyl)-1,3-butadiene (3k)

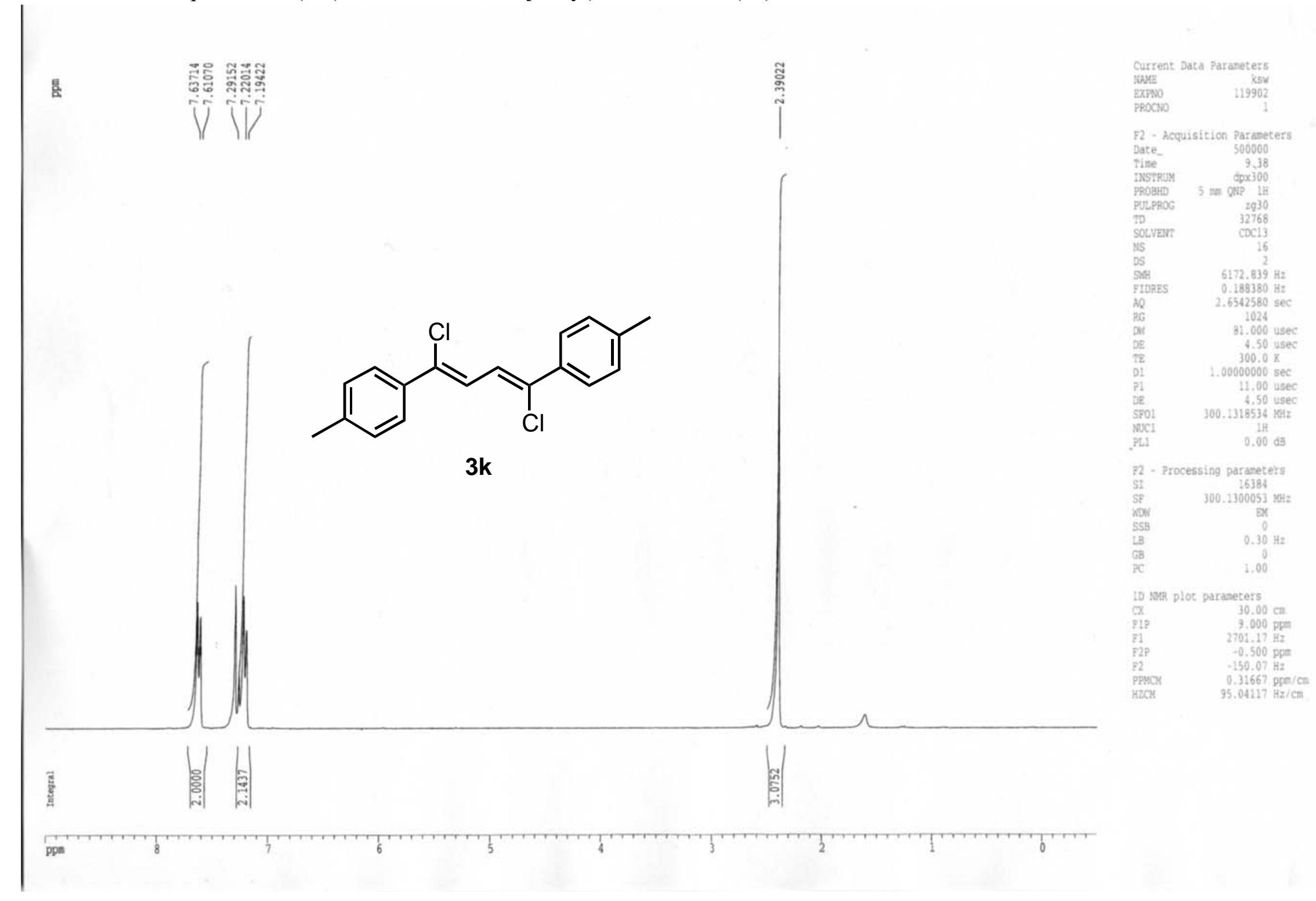


Appendix 26: ${ }^{13} \mathrm{C}$ NMR spectrum of (Z,Z)-1,4-dichloro-1,4-di(p-tolyl)-1,3-butadiene (3k)

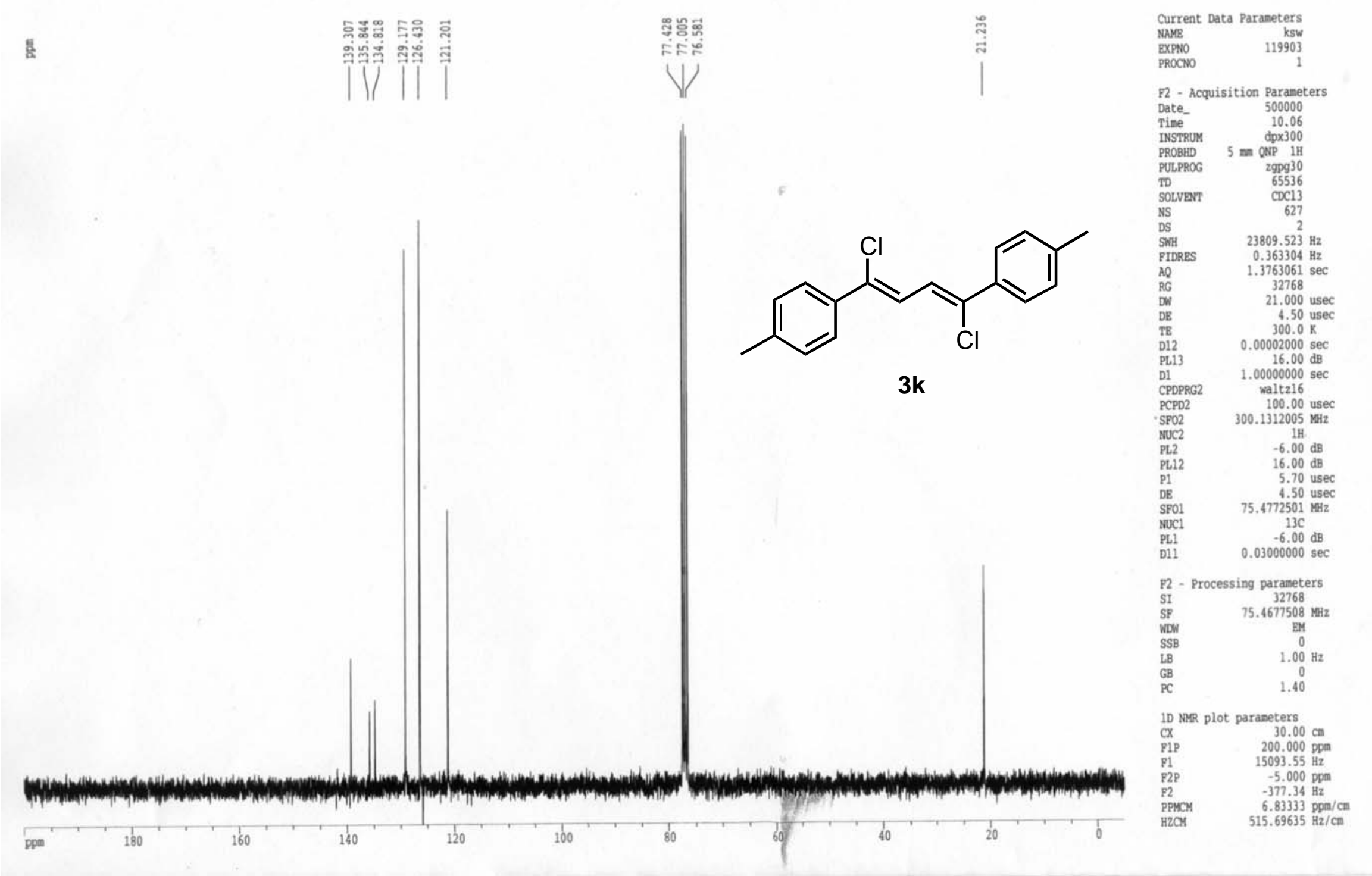

